\title{
Nanoporous and Nanostructured Aluminium and Titanium Oxide Thin Films
}

\author{
A. Froimovitch
}

A thesis submitted to the Faculty of Graduate Studies and Research in partial fulfillment of the requirements for the degree of Masters of Applied Science

\author{
Department of Electronics \\ Carleton University, \\ Ottawa, Ontario, Canada
}

September 2006

(C)Adam Froimovitch 


$\begin{array}{ll}\begin{array}{l}\text { Library and } \\ \text { Archives Canada }\end{array} & \begin{array}{l}\text { Bibliothèque et } \\ \text { Archives Canada }\end{array} \\ \begin{array}{l}\text { Published Heritage } \\ \text { Branch }\end{array} & \begin{array}{l}\text { Direction du } \\ \text { Patrimoine de l'édition }\end{array} \\ \begin{array}{l}\text { 395 Wellington Street } \\ \text { Ottawa ON K1A ON4 }\end{array} & \begin{array}{l}\text { 395, rue Wellington } \\ \text { Ottawa ON K1A ON4 } \\ \text { Canada }\end{array}\end{array}$

Your file Votre référence ISBN: 978-0-494-23336-8 Our file Notre référence ISBN: 978-0-494-23336-8

NOTICE:

The author has granted a nonexclusive license allowing Library and Archives Canada to reproduce, publish, archive, preserve, conserve, communicate to the public by telecommunication or on the Internet, loan, distribute and sell theses worldwide, for commercial or noncommercial purposes, in microform, paper, electronic and/or any other formats.

The author retains copyright ownership and moral rights in this thesis. Neither the thesis nor substantial extracts from it may be printed or otherwise reproduced without the author's permission.
AVIS:

L'auteur a accordé une licence non exclusive permettant à la Bibliothèque et Archives Canada de reproduire, publier, archiver, sauvegarder, conserver, transmettre au public par télécommunication ou par l'Internet, prêter, distribuer et vendre des thèses partout dans le monde, à des fins commerciales ou autres, sur support microforme, papier, électronique et/ou autres formats.

L'auteur conserve la propriété du droit d'auteur et des droits moraux qui protège cette thèse. $\mathrm{Ni}$ la thèse ni des extraits substantiels de celle-ci ne doivent être imprimés ou autrement reproduits sans son autorisation.
In compliance with the Canadian

Privacy Act some supporting forms may have been removed from this thesis.

While these forms may be included in the document page count, their removal does not represent any loss of content from the thesis.
Conformément à la loi canadienne sur la protection de la vie privée, quelques formulaires secondaires ont été enlevés de cette thèse.

Bien que ces formulaires aient inclus dans la pagination, il n'y aura aucun contenu manquant.

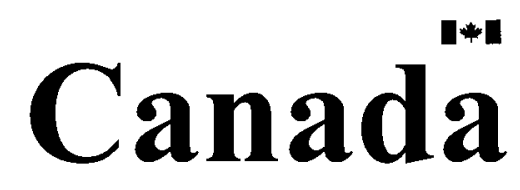




\section{Abstract}

Nanoporous alumina (NPA) and Nanostructured titania (NST) films are fabricated and characterized. The NPA is grown by anodic oxidation of aluminium metal. The structure and growing behavior of the NPA films are analyzed for a range of anodizing voltages. An accurate optical model is proposed for the NPA films over the entire range of voltages explored. The optical model is made to fit transparency data using the Levenberg Marquardt algorithm. The NPA is appropriate for optically -based sensing. The NST films are fabricated using a simple chemical oxidation technique and then analyzed for structure, stress, transparency, crystallinity and surface area. A well crystallized NST film with a specific surface area of $535 \mathrm{~m}^{2} / g$ is obtained by annealing the as-oxidized at films $240^{\circ} \mathrm{C}$. The low temperature anneal is compatible with roll to roll processing of dye sensitized solar cells or flexible electronics. 


\section{Acknowledgments}

I would like to thank my supervisor Professor Tom Smy, without whom none of this would have been possible. His guidance and support (both academically and financially) have been instrumental to my graduate and undergraduate experiences at Carleton University. I would like to express gratitude to Steven McGarry. His patience and diverse expertise have significantly added to my graduate studies here at Carleton. Other students, staff and faculty of Carleton University I would like to acknowledge for the help they gave me are Rob Vandusen, Carol Adams, Professor Garry Tarr, Dritan Celo, Peter Chyurlia, Harpreet Panesar, Tom Hewitt, Professor Nail Tait, Marsha Bissessarsingh, Dr. Paul Gammon and Tony O'Neil.

FE-SEM imaging and sample preparation for this work was performed in collaboration with Jason Apt and Chris Pawlowicz at Semiconductor Insights, and Jeff Fraser at the NRC-IMS. Surface area analysis of the NST films was performed with the help of Dr. Stephen Lang at the NRC-SIMS. ICP mass spectroscopy was performed by Dale Robertson at Paracel Labs.

Finally I would also like to thank Carol, Mark, Ira, Daniel and Michelle. Thank you for you love and support. 


\section{Contents}

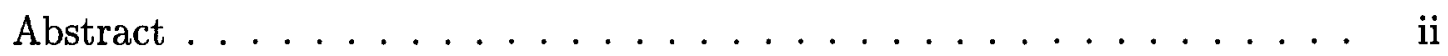

Acknowledgments . . . . . . . . . . . . . . . iii

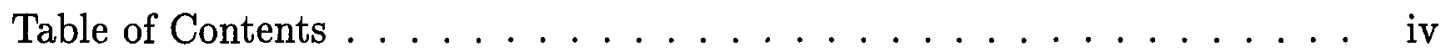

List of Tables $\ldots \ldots \ldots \ldots \ldots \ldots \ldots \ldots$ vii

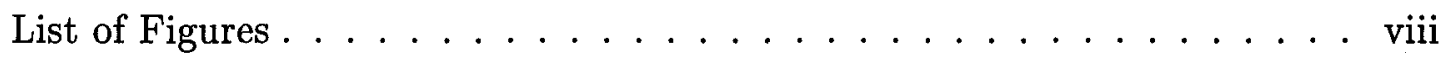

List of Abbreviations and Symbols $\ldots \ldots \ldots \ldots \ldots$ xi

1 Introduction 1

2 Fabrication of Nanoporous Alumina and Nanostructured Titania: $\begin{array}{ll}\text { Background } & 6\end{array}$

2.1 Nanoporous Alumina . . . . . . . . . . . . . . . . 6

2.1 .1 Oxide properties $\ldots \ldots \ldots \ldots \ldots \ldots$

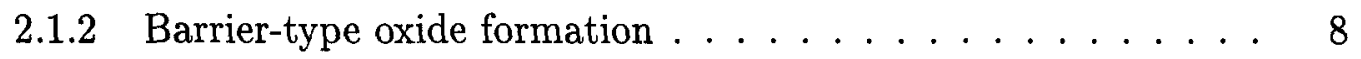

2.1.3 Porous-type oxide formation . . . . . . . . . . . 10

2.1.4 Discussion . . . . . . . . . . . . . . 13

2.2 Nanostructured Titania . . . . . . . . . . . . . . . . . 14

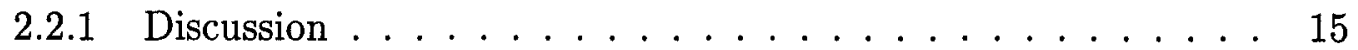

iv 
3 Nanoporous Alumina thin films: Fabrication and Characterization 17

3.1 Experimental Procedure .................... 18

3.1.1 Apparatus and film preparation ............ 18

3.1.2 Anodic current ................... 19

3.1.3 Optical characterization ................ 19

3.2 Results ......................... 23

3.2.1 Anodic Current ................... 24

3.2.2 Optical characterization .................. 24

3.3 Discussion . . . . . . . . . . . . . . . . . 26

3.4 Summary ............................ 29

4 Nanostructured Titania thin films: Fabrication and Characteriza$\begin{array}{ll}\text { tion } & \mathbf{4 0}\end{array}$

4.1 Experimental procedure . . . . . . . . . . . . . . 41

4.1 .1 NST preparation ................... 41

4.1 .2 Density calculation . . . . . . . . . . . . . . 42

4.1.3 Optical characterization .............. 43

4.2 Resutls . . . . . . . . . . . . . . . . . . 44

4.2.1 Film appearance and structure ............. 44

4.2.2 Film Stress and Cracking . . . . . . . . . . . 47

4.2.3 Film Surface Area and Porosity . . . . . . . . . . . . 50

4.2 .4 Transparency ..................... 53

4.2.5 Xray diffraction measurement . . . . . . . . . . . . 54

4.3 Summary ............................. 55 
$\begin{array}{ll}\text { Appendix A Levenberg-Marquardt algorithm } & 60\end{array}$

Appendix B LM Implementation $\quad 63$

B.1 Main program . . . . . . . . . . . . . . . . 63

B.2 evaluate ...................... 67

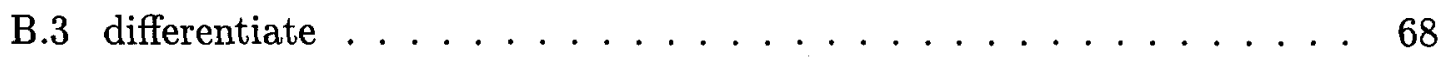

B.4 thinfilmTAl2 ...................... 69

$\begin{array}{ll}\text { References } & \mathbf{7 2}\end{array}$

vi 


\section{List of Tables}

4.1 Deflection of wafers due to titanium films deposited at different pressures 47

4.2 Surface area of NST normalized to $1 \mathrm{~m}^{2}$ of substrate. Pressure is measured in mTorr. .................... 51

4.3 Density measurements of NST film from parent Titanium film deposited at 25 mTorr on silicon substrate. All of the mass calculations are in grams and density is in $g / m^{3}$. Pressure and $V_{o x}$ are measured in $\mathrm{m}$ Torr and $\mathrm{m}^{3}$ respectively $\ldots \ldots \ldots$. . . . . . . . 52

vii 


\section{List of Figures}

1.1 (a) optical waveguide with a nanostructured core. (b) gas sensing device. 2

1.2 Figure (a) and (b) illustrate DSC operation and nanoparticulate film [1] respectively. The HOMO and LUMO state of the dye are denoted by

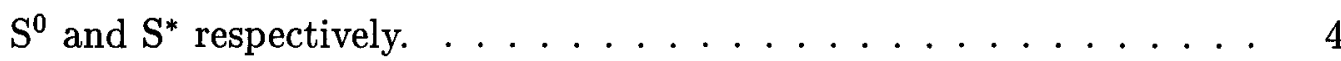

2.1 Anodization setup showing an aluminium anode connected through an electrolytic solution to a platinum cathode. When the power source is turned on the growth of a thick oxide takes place on the aluminium anode. An ammeter is placed in the circuit to measure anodic current. 8

2.2 Barrier-type anodic alumina. The arrow indicates the direction of the

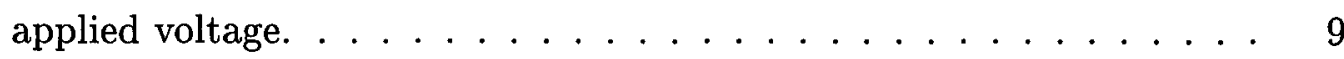

2.3 Porous-type anodic alumina: (a) the formation of a barrier type film with local variations in the thickness and density. (b) the variations in oxide properties result in regions of local high electric field/current density and increased etch rate. (c) the steady state appearance of the anodic alumina $\ldots \ldots \ldots \ldots \ldots \ldots \ldots$

2.4 Images of NPA grown through anodic oxidation of aluminium. Images (a) and (b) are taken from [2] and [3] respectively. . . . . . . 12 


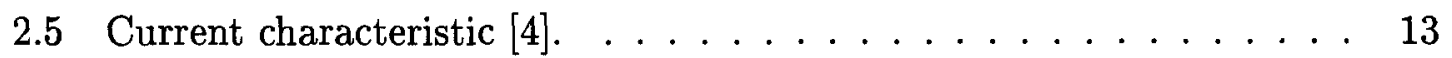

2.6 Hydrogen peroxide derived NST $[5] \ldots \ldots \ldots \ldots \ldots$

3.1 Digital photo of the first anodization apparatus used $\ldots \ldots \ldots$

3.2 (a) schematic diagram of the anodization apparatus. (b) close up digital photo of the anodization apparatus. (c) digital photo of entire apparatus including the power source $\ldots \ldots \ldots 20$

3.3 Current to voltage converter for measuring anodic current $\ldots \ldots .21$

3.4 (a) spectrophotometer used for reflectometry and (b) polycarbonate box: monochomator light passes though lens 1 , lens 2 , and then through the sample holder to the detector. . . . . . . . . . . . 22

3.5 Optical model of porous alumina films . . . . . . . . . . . . 24

3.6 Alumina films anodized at $70 \mathrm{~V} \ldots \ldots \ldots \ldots \ldots \ldots$

3.7 Inverse of etch rate vs applied voltage for $30-90 \mathrm{~V} \ldots \ldots \ldots 28$

3.8 Anodic current for a $30 \mathrm{~V}$ anodization. . . . . . . . . . . 29

3.9 Refractive index of glass substrate $\ldots \ldots \ldots \ldots \ldots \ldots$

3.10 Simple optical models for alumina films. Figure (a) is the result of the simplest model, pure oxide on a glass substrate. Figure (b) is the result of the next simplest model, pure oxide on a thin layer of pure metal on a glass substrate. . . . . . . . . . . . . . 31

3.11 Measured and modeled data for alumina film anodized at 50V . . . . 32

3.12 Refractive index for alumina films anodized between 30 and 90V. . . 33

3.13 Film thickness resulting from $500 \mathrm{~nm}$ of $\mathrm{Al}$ and scattering coefficient for films anodized between 30 and $90 \mathrm{~V} \ldots \ldots \ldots \ldots \ldots$

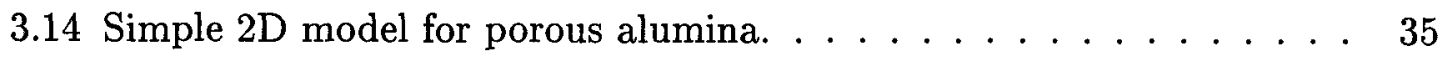


3.15 Alumina film anodized at 30V under high and low magnification. . . 37

4.1 SEM images of titanium films a) before and b) after oxidation . . . 42

4.2 Top surface of NST derived from Titanium film deposited at $25 \mathrm{mTorr}$ and oxidized for 45 minutes in $\mathrm{H}_{2} \mathrm{O}_{2} \ldots \ldots \ldots \ldots \ldots \ldots$

4.3 FE-SEM cross-section of NST showing the porous layer on top, an intermediary titania water complex layer and the remaining metal film. 45

4.4 FT-IR measurements indicating $\mathrm{OH}$ bond stretching. . . . . . . . . 46

4.5 Mechanically polished cross-sections of (a) as oxidized and (b) annealed NST. The intermediary layer is more compact after annealing 47

4.6 Bending of substrate due to titanium film on silicon wafer . . . . . 47

4.7 FE-SEM images of NST surface for different titanium sputtering pressures (a) 2.5 mTorr, (b) 8 mTorr and (c) 25 mTorr respectively. A $10 \mu \mathrm{m}$ bar is shown in each. . . . . . . . . . . . . . 48

4.8 BET nitrogen isotherms. Sputtering pressure (a) $2.5 \mathrm{mTorr}$, (b) 8 mTorr, (c) 25 mTorr, d) 25 mTorr annealed. . . . . . . . . . . . . 50

4.9 Polished FE-SEM sections of a) parent metal film, b) remaining metal film after oxidation and c) resulting NST . . . . . . . . . . 51

4.10 Transparency of annealed (bare) and dyed NST films compared to

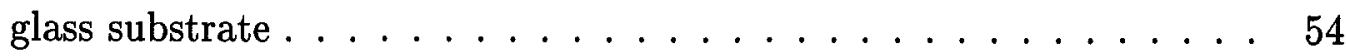

4.11 XRD pattern of (a) as-oxidized, (b) NST annealed for 24 hours at $240^{\circ} \mathrm{C}$ and $\left(\right.$ c) 10 hours at $300^{\circ} \mathrm{C} \ldots \ldots \ldots \ldots$ 


\title{
List of Abbreviations and Symbols
}

\author{
$\alpha \quad$ Proportionality constant \\ $\beta \quad$ Proportionality constant \\ $\lambda \quad$ Wavelength \\ $\rho \quad$ Density \\ $\rho_{p} \quad$ Density of porous material \\ $\rho_{o x} \quad$ Oxide density \\ $A \quad$ Area \\ $M \quad$ Mass \\ $M M_{o x}$ Molar mass oxide \\ $M M_{T i}$ Molar mass Ti \\ $r_{p} \quad$ pore radius \\ $t \quad$ Thickness \\ $t_{p} \quad$ pore spacing \\ $T_{o x} \quad$ Oxide thickness \\ $V \quad$ Voltage \\ $V_{c} \quad$ Volume of unit cell \\ $V_{p} \quad$ Pore volume \\ $V_{o x} \quad$ Oxide volume
}


$\mu \mathrm{m} \quad$ micron

DSC Dye Sensitized Solar Cell

EMA Effective Medium Approximation

FE-SEM Field Emission Scanning Electron Microscope

FT-IR Fourier Transform Infrared Spectroscopy

HOMO Highest Occupied Molecular Orbital

ICP Inductively Coupled Plasma

IR Infrared

ITO Indium tin oxide

k Extinction coefficient

LM Levengerg Marquardt

LMS Least Mean Square

LUMO Lowest Unoccupied Molecular Orbital

$\mathrm{N}$ Complex index of refraction

n Refractive index

nm nanometer

NPA Nanoporous Alumina

NST Nanostructured Titania

P Porosity

SEM Scanning Electron Microscope

SSA Specific Surface Area

UV Ultraviolet

VIS Visible

xii 


\section{Chapter 1}

\section{Introduction}

In recent years, the growth, characterization and potential use of nanostructured materials $[4,5,6,7,8,9,10]$ have caught the attention of many researchers in both industry and academia. Nanostructured materials (nano-materials) are those which exhibit a large degree of porosity and or structure on the nanometer scale. The large surface area and pore volume characteristic of these materials combined with either conducting, semiconducting or dielectric properties result in diverse applications such as sensing, energy production and pollution management $[7,11,12,13,14]$. Nanostructured thin films are of particular interest. Thin films are typically on the order of $1 \mu \mathrm{m}$ thick and are easily integrated into current microelectronics fabrication technologies. They can be produced on flexible substrates and have the potential for roll to roll processing.

A wide range of applications exist for nanostructured thin films. Nanoporous dielectric and semiconducting thin films have been shown to be effective and extremely sensitive for sensing applications [11]. The unique dimensionality of these materials 
allows them to interact with their surroundings to a degree which is difficult for non-porous or non-structured materials. Figure 1(a) and (b) provide examples of some nanostructured sensing devices found in the literature. Figure 1(a) shows a nanostructured dielectric forming the core of an optical waveguide. Light traveling through this waveguide will see the nanoporous dielectric and the material penetrating its pores as one effective medium with well defined optical constants $[8,11,15,16]$. Small changes in the refractive index of the penetrating material can be detected as a change in the effective index of the waveguide core. Such a device was produced using nanoporous alumina (NPA) films in [11], and used to monitor changes in the structure of the NPA in-situ. Figure 1(b) shows two metal contact pads separated by a nanostructured wide bandgap semiconductor. At room temperature the wide bandgap semiconductor has few free carriers, and therefore does not conduct. However, when certain gases penetrate the nanostructured semiconductor gas molecules bond to the surface creating vacancies and surface states, effectively doping the surface and increasing the conductivity. If the surface area of the semiconductor is significant, then the change in conductivity will be measurable. Such a device was fabricated from nanostructured titania (NST) for oxygen sensing in [4].

Nanostructured semiconductors have been found to be extremely useful in the production of clean renewable energy and in the oxidation of pollutants. Once again, the large surface area or pore volume of such materials allows for efficient light harvesting in dye-sensitized solar cells (DSC) $[1,7,17]$, a relatively new and cost effective alternative to conventional PN junction solar cells, as well as in the production of hydrogen or oxidation of pollutants through photocatalysis $[12,13,14]$.

Figure 1.2(a) illustrates the basic operation of a DSC. Light enters the cell through 


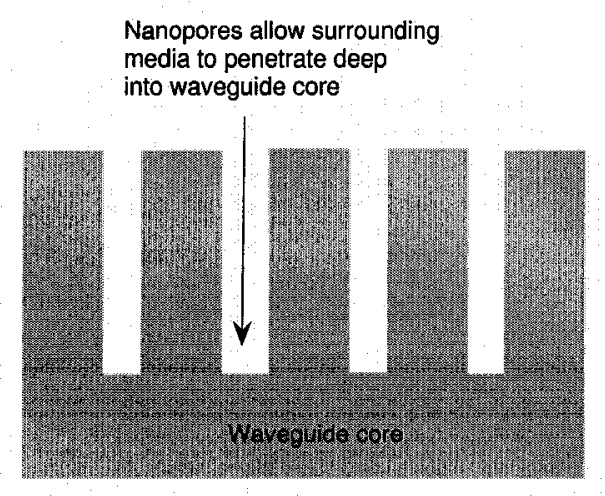

(a)

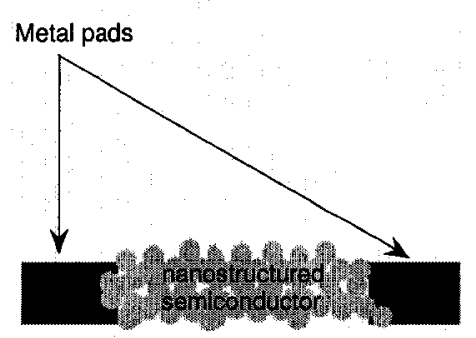

(b)

Figure 1.1: (a) optical waveguide with a nanostructured core. (b) gas sensing device.

the back side of a nanostructured electrode, typically NST. A dye monolayer is bonded to the surface of the NST. Light is absorbed by dye molecules by promotion of an electron from the highest occupied molecular orbital (HOMO) to the lowest unoccupied molecular orbital (LUMO). The promoted electron is transferred from the LUMO state of the dye, to the conduction band of the NST, leaving the dye oxidized. A transparent conductor such as indium tin oxide (ITO) collects the electrons injected into the NST from the dye, which is subsequently reduced by a redox system inside the solar cell $[1,7]$. Figure 1.2(b) shows a typical NST electrode for DSCs. This type of NST electrode is formed by sintering a nanoparticulate slurry of anatase and rutile at $600{ }^{\circ} \mathrm{C}[7]$.

Key to the integration of nano-materials into working optical or electronic sensors, 


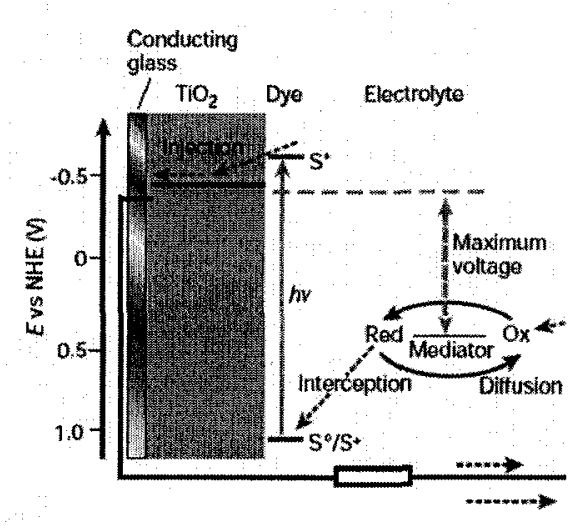

(a)

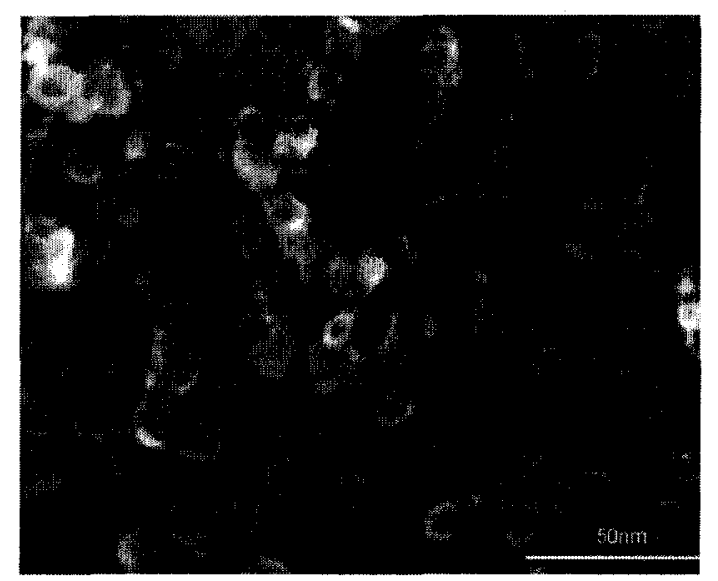

(b)

Figure 1.2: Figure (a) and (b) illustrate DSC operation and nanoparticulate film [1] respectively. The HOMO and LUMO state of the dye are denoted by $\mathrm{S}^{0}$ and $\mathrm{S}^{*}$ respectively.

solar cells or photocatalysts, are methods for quantifying their unique properties. In the case of optical sensors accurate optical models are required to extract the optical parameters such as the refractive index and extinction coefficient. These models are often quite complex, as a result of the porous nature of nano-materials. In the case of solar cells, photocatalysts or semiconducting sensors properties such as crystallinity, surface area and electron mobility are of importance. Critical to all such devices, is the ability to control or at least verify the dimensions and characteristics of the nanostructure. In sensing devices, the physical structure will be instrumental in obtaining an accurate optical model. For semiconducting devices, such as solar cells or photocatalysts the physical structure of the device can greatly effect the efficiency of electron transport. Highly ordered and well connected nanostructured electrodes are thought to be better electron transporters than the sintered nanoparticulate films 
shown in Fig. 1.2(b). A key point in DSC research has then become the ability to control and monitor the structure of NST electrodes.

The following work presents a detailed analysis on the characterization of some key properties of nano-materials described in the preceding paragraph. Two types of nanostructured thin films are fabricated using simple electrochemical and chemical means. The first material explored is NPA which is fabricated through the anodic oxidation of aluminium films deposited on glass $[2,18]$. The physical structure and growth parameters of the NPA are explored for different anodizing conditions using a variety of techniques. Several optical models are proposed to accurately match measured transparency data obtained from NPA grown under slightly different anodizing conditions. The Levenberg Marquardt (LM) algorithm is used to extract the optical constants of the NPA under these growing conditions.

The second type of material explored is NST which is fabricated using a chemical oxidation technique first proposed in [19]. Electron microscopy is used to verify that the NST fabricated is a highly ordered and well connected network for enhanced electron transport. Other key properties of the NST are also explored, such as surface area, transparency and crystallinity.

The remainder of this work will be divided into 4 chapters. Chapter 2 provides the background and theory required to understand the fabrication techniques behind the nano-materials discussed here. Chapter 3 and 4 provide detailed analysis of the fabrication and characterization of the NPA and NST described in the preceding paragraph respectively. Finally, chapter 5 provides a summary and conclusion for the entire work. 


\section{Chapter 2}

\section{Fabrication of Nanoporous}

\section{Alumina and Nanostructured}

\section{Titania: Background}

This chapter provides a brief introduction to the fabrication techniques used to produce NPA and NST. NPA films are produced through the anodic oxidation of aluminium thin films, a simple electrochemical process $[3,9,10,16,18,2,20]$. NST films are produced though a chemical oxidation technique following works presented in $[4,5,19]$.

\subsection{Nanoporous Alumina}

It has been established for some time that a high surface area and highly ordered porous amorphous aluminum oxide film can be formed by the anodization of aluminum in some acidic media $[2,3,9,10,16,18,20]$. Recently these alumina films 
have been used as nanostructured templates for the fabrication of other porous materials such as gold $[8,15]$ and titania $[12,13]$. Of interest here are the optical properties, physical structure and growth characteristics (growth rate) of NPA. If NPA is to be incorporated into optically based sensors, then an accurate optical model is required to extract the refractive index and extinction coefficient. Information on the physical structure and growth characteristics can be correlated to the optical model to provide a measure of control over the refractive index and extinction coefficient.

Anodization is a process were the native oxide on some metals, including aluminium, can be enhanced electrochemically. The enhanced oxide layer is typically thicker than the native layer and inhibits corrosion or scratching of the metal beneath. Figure 2.1(a) shows a typical set up for the anodization of aluminium. An aluminium sheet or deposited film is made to be the anode of an electrochemical cell and a platinum gauze or sheet is used as the cathode. In between the anode and cathode is an aqueous electrolyte. The electrolyte is typically a weak acid or base, and its properties can significantly affect the properties of the enhanced oxide. When a positive voltage is applied to the anode with respect to the cathode the electrolyte facilitates hydrolysis of aluminium according to

$$
3 \mathrm{H}_{2} \mathrm{O}+2 \mathrm{Al} \rightleftharpoons \mathrm{Al}_{2} \mathrm{O}_{3}+3 \mathrm{H}_{2}
$$

The hydrogen appears as bubbles of gas at the platinum electrode. Equations 2.1 presents a simplified model for the electrochemistry, and is the main driving force of the anodization process. In reality however, more complex reactions including the electrolyte anion are also involved [16]. 


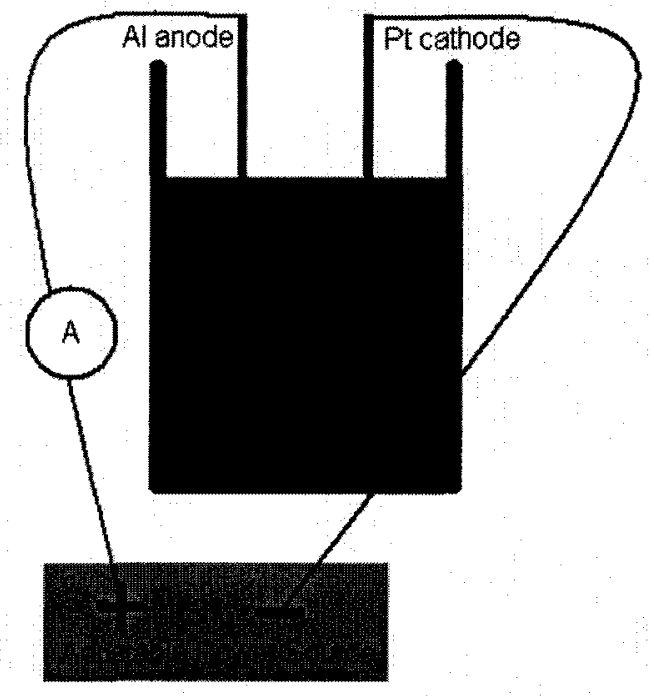

Figure 2.1: Anodization setup showing an aluminium anode connected through an electrolytic solution to a platinum cathode. When the power source is turned on the growth of a thick oxide takes place on the aluminium anode. An ammeter is placed in the circuit to measure anodic current.

\subsubsection{Oxide properties}

The properties of the anodic oxide vary greatly with the $\mathrm{pH}$ of the electrolyte. A high $\mathrm{pH}$ (basic solution) results in a dense barrier-type film. A low pH (concentrated acid) will result in the complete dissolution of the anodic oxide. A medium to low $\mathrm{pH}$ (dilute acid solution) will result in a highly ordered porous oxide. 


\subsubsection{Barrier-type oxide formation}

A barrier-type oxide layer will develop on aluminium when it is anodized in a basic electrolyte, such as $\mathrm{NaOH}$. When a DC voltage is applied to the apparatus in Fig. 2.1 an insoluble anodic oxide layer begins to develop on the surface of the aluminium layer. As the reaction proceeds, hydroxide ions are driven by the electric field through the oxide layer to the metal where they react to produce more oxide. This reaction will continue until the oxide layer becomes too thick for the hydroxide ions to penetrate. The resulting anodic oxide is therefore a dense barrier layer, the thickness of which is directly proportional to the applied voltage [10]. Figure 2.2 graphically depicts the growth and structure of a barrier-type anodic aluminium oxide.

\subsubsection{Porous-type oxide formation}

A porous-type oxide layer will develop on aluminium when it is anodized in a weak acidic solution, such as dilute $\mathrm{H}_{2} \mathrm{SO}_{4}$. When the anodization begins, a thin barriertype layer develops on the surface of the metal. As the reaction proceeds, nonuniformities in the electric field and current density penetrating the barrier film result in preferred etching of the oxide in regions of increased electric field or current

density [16]. The sources of these non-uniformities are not well understood, but may be caused by imperfections in the surface structure and variations in the density of the oxide inherited from the aluminium anode. As the reaction proceeds deep pores start to form in these regions, resulting in a porous oxide [16]. 


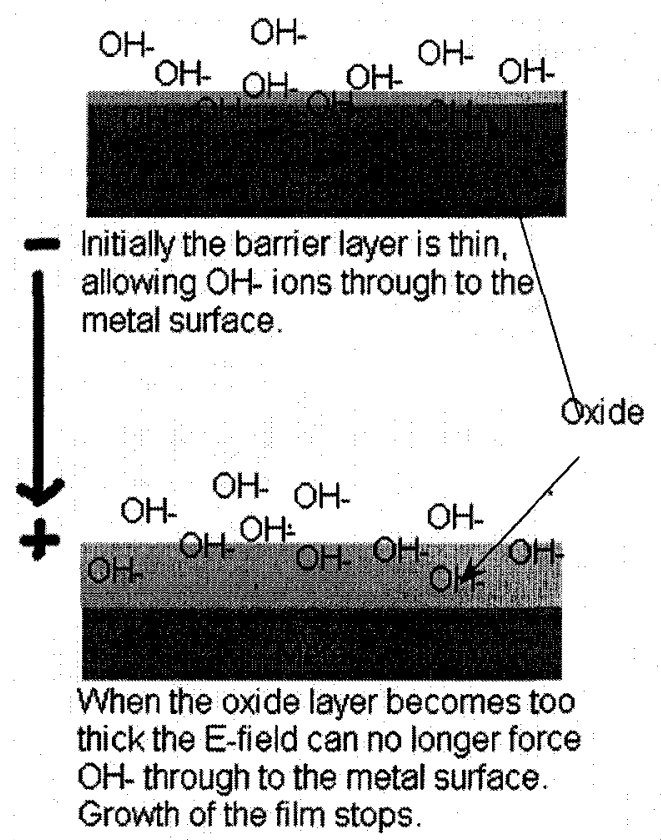

Figure 2.2: Barrier-type anodic alumina. The arrow indicates the direction of the applied voltage. 


\section{Structure and growth}

Figure 2.3 illustrates the structure and growth of porous anodic alumina. Figure 2.3(a) shows the initial barrier-type film, which has inherited some thickness and density variations possibly from the parent metal film. Figure 2.3(b) illustrates how these local variations in thickness and density result in local enhancement of the electric field and the initiation of pores. The steady state growth of the porous oxide is illustrated in Fig. 2.3(c).

Figures 2.4(a) and (b) are of field emission scanning electron microscope (FESEM) images of anodic NPA taken from [2] and [3] respectively. The images show the structure of a porous anodic alumina film from the top surface and in crosssection. Studies have shown that the pore radius and the wall are proportional to the anodizing voltage $[3,9,16]$. The proportionality constants are typically functions of the electrolyte and the temperature. Following $[3,9]$ the pore radius and wall thickness can be expressed as $r_{p}=\alpha V$ and $t_{p}=\beta V$ respectively, where $r_{p}$ is the pore radius, $t_{p}$ is the pore spacing, $\alpha$ and $\beta$ are proportionality constants. Figure 3.14 graphically illustrates the pore spacing $t_{p}$ and radius $r_{p}$. 


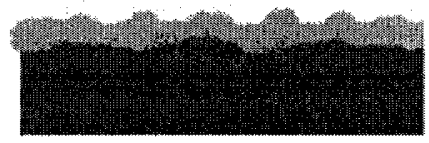

a) Local variations in oxide thickness

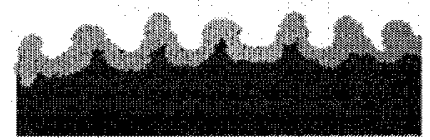

b) Increased E-field and oxide etch

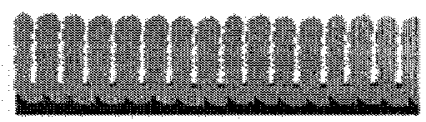

c) Steady state growth

Figure 2.3: Porous-type anodic alumina: (a) the formation of a barrier type film with local variations in the thickness and density. (b) the variations in oxide properties result in regions of local high electric field/current density and increased etch rate. (c) the steady state appearance of the anodic alumina 


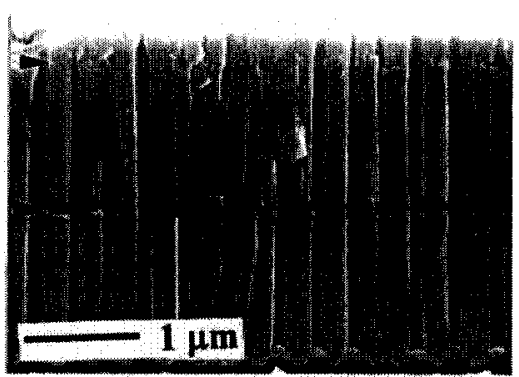

(a) side view

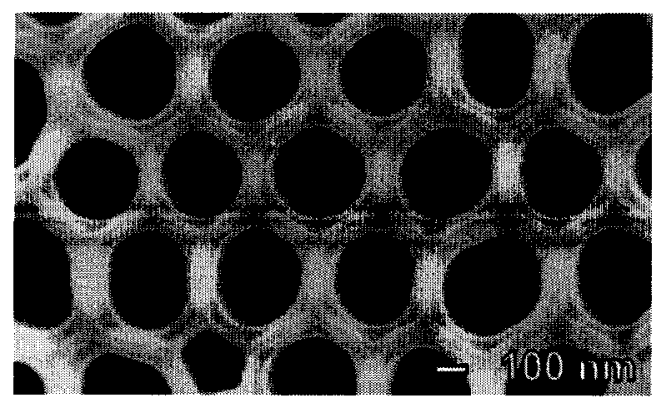

(b) top view

Figure 2.4: Images of NPA grown through anodic oxidation of aluminium. Images (a) and (b) are taken from [2] and [3] respectively.

\section{Anodic current}

Figure 2.5 shows a typical current-time behavior for the anodization of a porous anodic alumina film [4]. The initial behavior is dominated by a decaying exponential. This represents the formation of the barrier layer. As the barrier layer becomes thicker, the flux of hydroxide ions which are able to penetrate gets smaller therefore resulting in a decaying current. At a certain instance, anodic current reaches a minimum and then increases. This is the onset of local oxide dissolution, resulting in an increase in anodic current. The plateau which follows is the steady state growth of the porous anodic oxide [18]. The final cut off in anodic current is due to the complete oxidation of all available aluminium. 


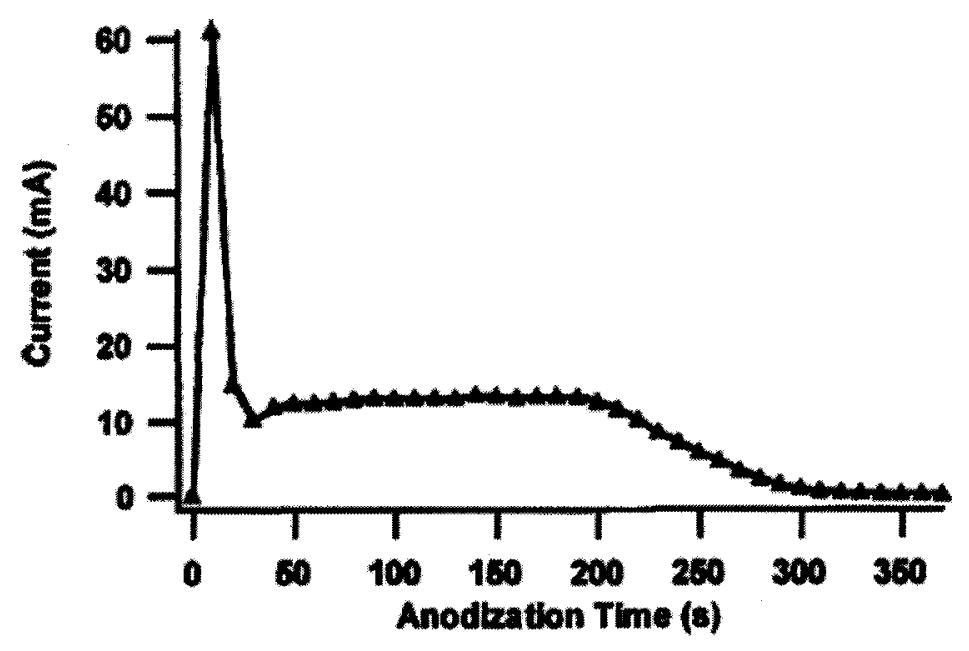

Figure 2.5: Current characteristic [4].

\subsubsection{Discussion}

Highly ordered NPA films can be fabricated through the anodic oxidation of aluminium metal in weakly acidic solutions. The literature has shown that the pore radius and pore spacings can be controlled by the anodizing voltage [3, 9].

The usefulness of anodically grown NPA as an optically based sensor has been demonstrated in the literature as described in chapter 1. Work presented in [11] demonstrates that the modes of an optical waveguide similar to Fig. 1(a) with either an NPA core or cladding can be extremely sensitive to the refractive index of the surrounding materials. The authors of [11] present single wavelength measurement of 
the effective permittivity of the anodic NPA used in their experiments. Earlier studies have also attempted to approximate the refractive index and extinction coefficient of anodic NPA and anodic NPA-gold composites using effective medium approximations (EMA). One approach taken by some has been to combine tabulated values of the constituent materials using an EMA such as Bruggemann, see chapter 3 [8]. Others have tried extract values for $n$ and $k$ at selected wavelengths using an analytic expression for the transmittance of NPA films [15].

One method that is often used by researchers when attempting to characterize the optical parameters of a thin film is to use spectroscopic reflectometry [21]. This approach involves performing a full non-linear regression on the least mean square error between measured optical data and an optical model of the refractive index and extinction coefficient. A more detailed explanation is given in Sec. 3.1.3 and 3.1.3.

A full spectroscopic analysis of the optical parameters would result in a dispersion model of the NPA for the IR-VIS-UV spectrum. The dispersion model can be compared to the constituent bulk materials, and to infer information on the structure and composition of the NPA. Furthermore, the accuracy of the extracted optical constants could be verified by the goodness of fit between the model and the actual measured data[21]. Such a spectroscopic analysis of porous alumina could not be found in the literature, and is presented in chapter 3.

\subsection{Nanostructured Titania}

A variety of methods exist for the production of nanostructured titania films. NST has been fabricated using the sol-gel method [22], through the anodic oxidation of 
titanium metal $[23,24,25]$ and by sintering colloidal nanoparticulate anatase/rutile mixtures. In recent years NST has become of particular interest as a wide bandgap semiconductor for sensing and photovoltaic applications, specifically DSCs. For many of these applications, particularly in DSCs, highly ordered NST films are preferred because they are thought to result in more efficient electron transport [17]. Furthermore, the in-situ formation of low temperature NST thin films is desirable for both photovoltaic and sensing applications because of the potential for flexible substrates and roll to roll processing.

An interesting study on NST films [19] demonstrated that in-situ processed amorphous NST films could be obtained by reacting titanium sheets with $10 \%$ hydrogen peroxide heated to $80^{\circ} \mathrm{C}$. The amorphous films could be transformed into a crystalline phase by annealing at a temperature of $300^{\circ} \mathrm{C}$ for 1 hour. It was reported that extended oxidation times (greater than 1 hour) resulted in the titania films cracking and delaminating from the titanium substrate $[4,5,19]$.

Further studies $[4,5]$ reported on the production NST by reacting titanium thin films deposited by electron beam evaporation onto thermally grown $\mathrm{SiO}_{2}$ with hydrogen peroxide in a similar manner to [19]. As concluded in $[4,5]$ NST cracking was due to tensile forces caused by shrinkage of the film upon removal from the hydrogen peroxide solution. By pre-patterning the original Titanium films into patches of $20 \mu \mathrm{m}$ cracking could be completely eliminated and large arrays of NST patches could be produced on a silicon substrate.

Figure 2.6 shows an FE-SEM image of hydrogen peroxide grown NST on thermally grown $\mathrm{SiO}_{2}$, where Figs.(a), (c) and (d) show images of NST which has cracked 


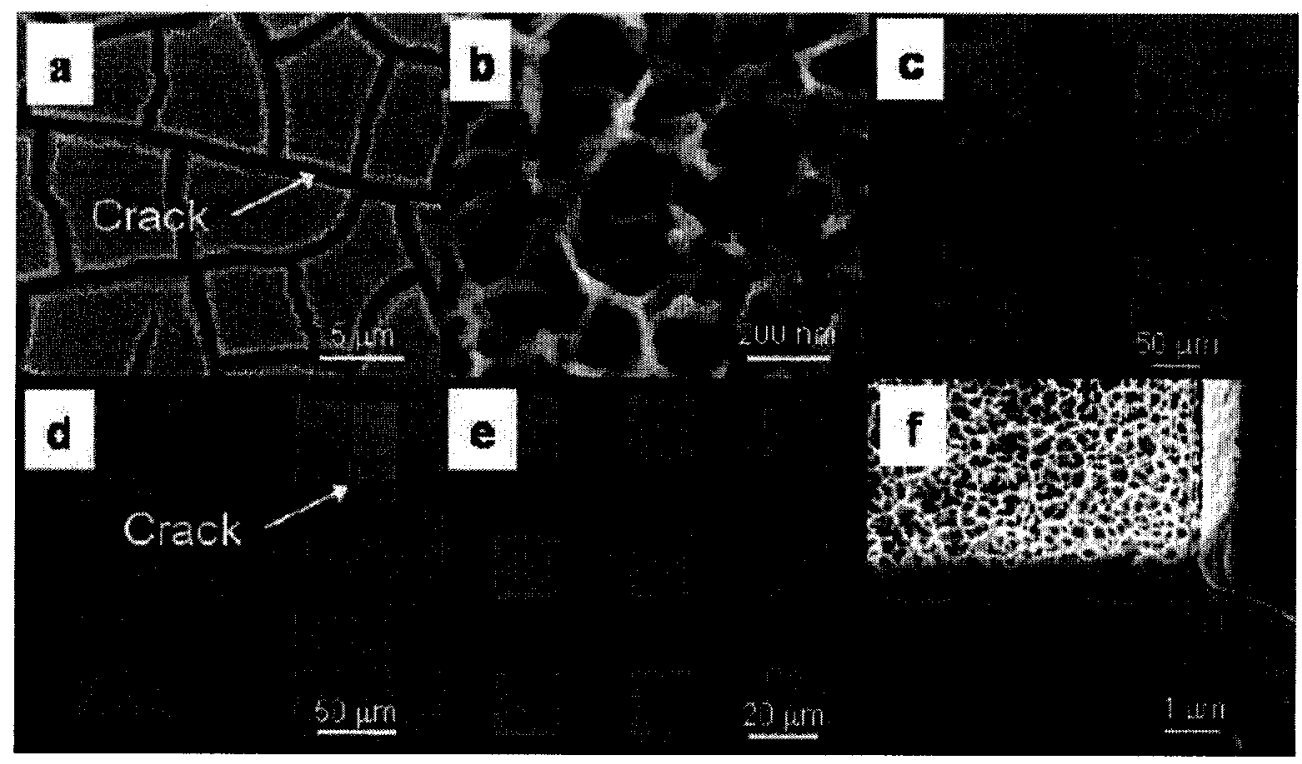

Figure 2.6: Hydrogen peroxide derived NST [5]

due to an extended oxidation time. Figures (e) and (f) are of their patterned Titanium substrates which result in crack free NST $[4,5]$.

\subsubsection{Summary}

Highly porous, well connected and ordered NST films can be obtained by a simple chemical oxidation process $[4,5,19]$. Extended oxidation times result in the NST cracking and delaminating from the substrate. It has been demonstrated that prepattering the NST into $20 \mu \mathrm{m}$ pads results in crack free arrays. The usefulness of the chemically grown NST arrays has been demonstrated in gas sensing and biomedical applications $[4,5]$. 
The potential application of hydrogen peroxide grown NST in dye-sensitized solar cells or as a photo-catalyst is not discussed in the literature. Information concerning the surface area and transparency of the NST, which would be critical for its use in either DSC or photocatalysts, is not presented anywhere in the literature. If it can be demonstrated that the hydrogen peroxide grown NST does have a large surface area and good transparency, then its potential use in DSCs should be considered 


\section{Chapter 3}

\section{Nanoporous Alumina thin films: Fabrication and Characterization}

In this section the structural and optical properties of anodic alumina formed at low anodization voltages are explored. An apparatus is constructed for producing porous anodic alumina films like those described in the previous chapter. Aluminium films deposited by electron beam evaporation are anodized in $5 \%$ phosphoric acid. Scanning electron microscopy confirms that alumina films anodized are indeed highly ordered and porous. The reaction rate and oxide thickness are both recorded as a function of anodization voltage. An optical model is presented which accurately describes the refractive index and extinction coefficient of the porous alumina films formed at low anodization voltages ( 30 to $90 \mathrm{~V}$ ). This optical model could be easily integrated to accurately predict the behavior of more complex optical structures and sensing devices. 


\subsection{Experimental Procedure}

The experimental procedure outlined in $[2,18]$ for anodic alumina fabrication was used as a guideline. $500 \mathrm{~nm}$ aluminium films were electron beam evaporated onto corning 7509 glass substrates. Platinum counter electrodes were fabricated by planar RF sputtering 100nm of platinum on Fisher microscope slides. A sputter deposited $50 \mathrm{~nm}$ layer of titanium used as an adhesion layer between the glass substrate and the platinum layer. Aluminium and platinum electrodes were made from these deposited films by cutting them into smaller squares or rectangles.

\subsubsection{Apparatus and film preparation}

To perform the anodization, a simple apparatus similar to the one shown in Fig. 2.1 was constructed. The apparatus consisted of a power source, a beaker, two alligator clips to connect the aluminum and platinum electrodes to the power source and an adjustable stand to hold the electrodes in place. The electrolyte (5\% phosphoric acid) was placed into the beaker so that the meniscus fell just below the alligator clips. A digital photo of the apparatus is shown in Fig. 3.1. This simple apparatus proved to be ineffective. At high voltages (greater than $50 \mathrm{~V}$ ) breakdown of either the oxide or the anodizing solution occurred at the air/acid/anode interface. It was hypothesized that the curvature of the meniscus at the aluminium anode resulted in a higher electric field and therefore dielectric/electrolytic breakdown. To circumvent this problem, a more sophisticated apparatus was constructed in which the meniscus did not fall on the aluminium anode. 


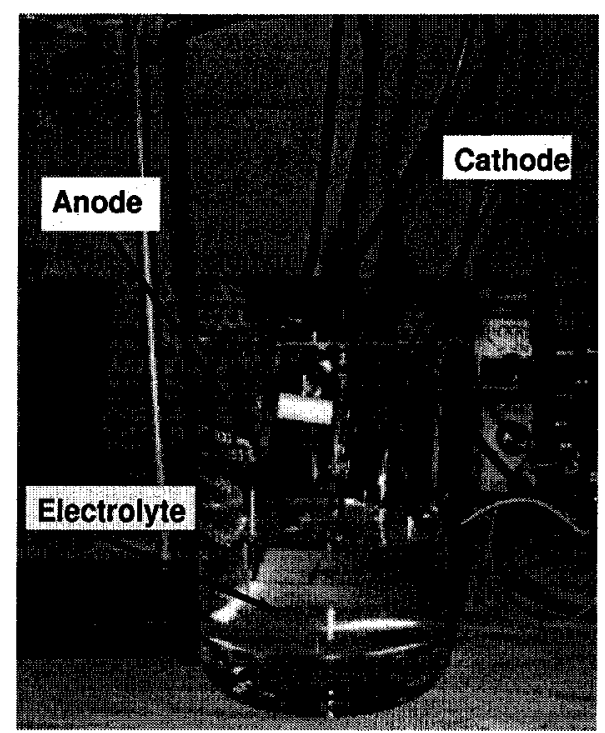

Figure 3.1: Digital photo of the first anodization apparatus used

The second apparatus consisted of an open polycarbonate tube and a polycarbonate sheet that were pressed together to form a vessel for the electrolyte. A Viton O-ring was placed between them and springs were used to clamp down on the Oring forming a water tight seal. An aluminium film on corning glass (the anode) was placed between the polycarbonate tube and the polycarbonate sheet. The Oring separated the aluminium film on glass from the end of the polycarbonate tube. Screws were used to clamp the springs downwards until the O-ring formed a water tight seal between the tube and the aluminium film. A schematic diagram and a digital photo is are shown in Fig. 3.2(a) and (b). A digital photo of the entire apparatus including the voltage source and ammeter is shown in Fig. 3.2(c). With this apparatus anodization voltages as high as $100 \mathrm{~V}$ could be attained.

With the improved apparatus aluminium films were anodized at 30, 40, 50, 55, 


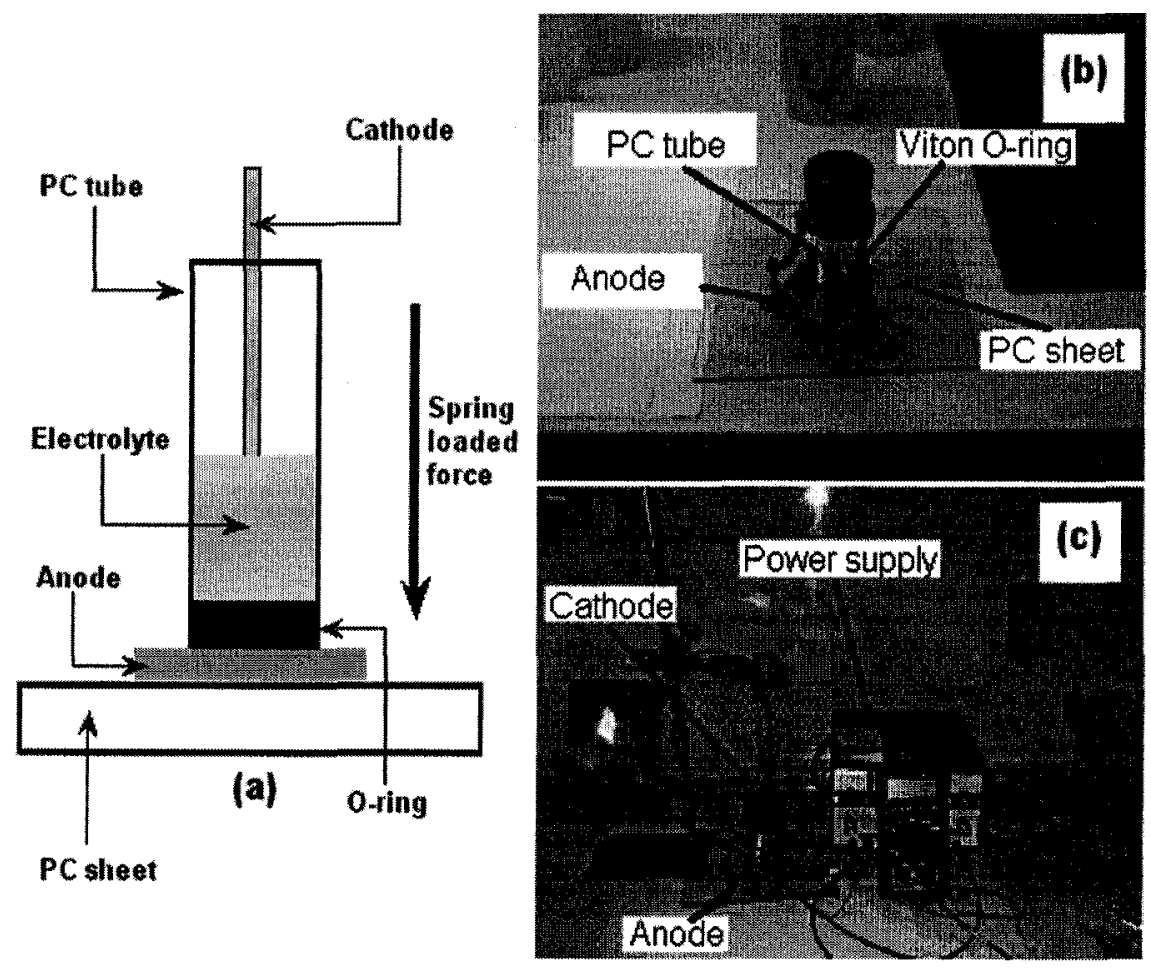

Figure 3.2: (a) schematic diagram of the anodization apparatus. (b) close up digital photo of the anodization apparatus. (c) digital photo of entire apparatus including the power source 
70,80 and 90 volts until the film became transparent, indicating the complete consumption of the aluminium metal. The reaction time until completion and the oxide thickness were recorded for each voltage. A Tencor P1 long scan profiler was used to measure the step height from the top of the oxide to the glass substrate.

\subsubsection{Anodic current}

The current to voltage amplifier shown in Fig. 3.3 was placed in series with the power source and the cathode in order to measure the anodic current. The output of the current to voltage amplifier was recorded by a laptop PC using a Labjack U12 analog to digital converter [26].

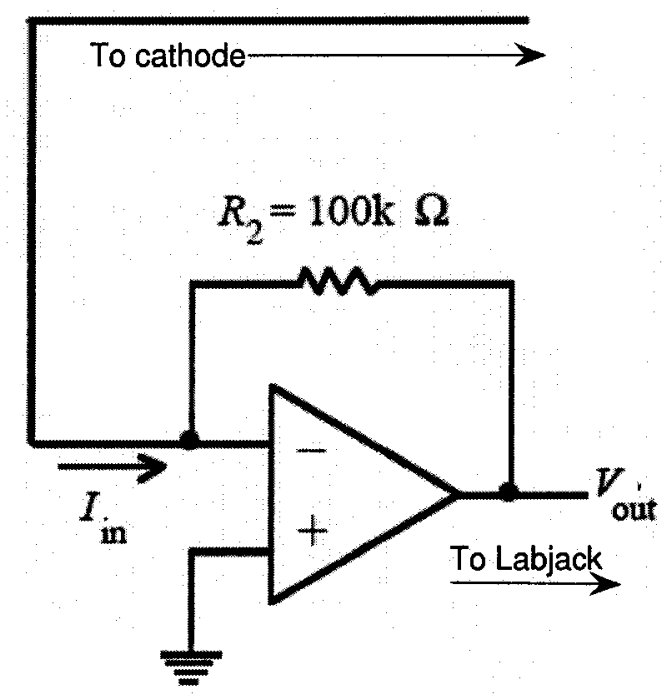

Figure 3.3: Current to voltage converter for measuring anodic current 


\subsubsection{Optical characterization}

A spectrophotometer was constructed and used to measure the transmittance of the anodized films on glass substrates. Light from an Oriel $6333100 \mathrm{~W}$ quartz tungsten halogen bulb housed in the Oriel 7340 Monochromator Illuminator was filtered using an Oriel 74000 Cornerstone 130 motorized monochromator. Monochromator light was focused by a lens onto the substrate. An Oriel M70311 silicon detector behind the transparent substrate measured the intensity of light on the other side of the substrate and delivered the data to an Oriel 70310 Multifunctional Optical Power Meter. Oriel Tracq32 V3.1 software was used to computer control the output spectra of the monochromator and intensity was recorded as a function of wavelength. A polycarbonate box painted black housed the focusing lenses and sample holder to keep ambient light out of the detector during measurements. Figure 3.4(a) presents a digital photograph of the spectrophotometer used. Figure 3.4(b) illustrates the inside of the polycarbonate box, where the lenses and sample holder were contained.

Once the transmittance of the alumina films was obtained, the LM algorithm was used to minimize the least mean square error (LMS) between the optical model, see section 3.1.3, and the measured data [21]. The LMS is given by

$$
L M S=\frac{1}{N-A} \Sigma\left[\frac{x_{n}-x_{n}^{\prime}}{\sigma_{n}}\right]^{2}
$$

where $x_{n}$ is the $\mathrm{n}^{\text {th }}$ measured data point, $x_{n}^{\prime}$ is the $\mathrm{n}^{\text {th }}$ model data point and $\sigma_{n}$ is the standard deviation of the $\mathrm{n}^{\text {th }}$ data point. $N$ is the number of data points and $A$ is the number of parameters in the optical model. The LM algorithm presented in Appendices A and B. 

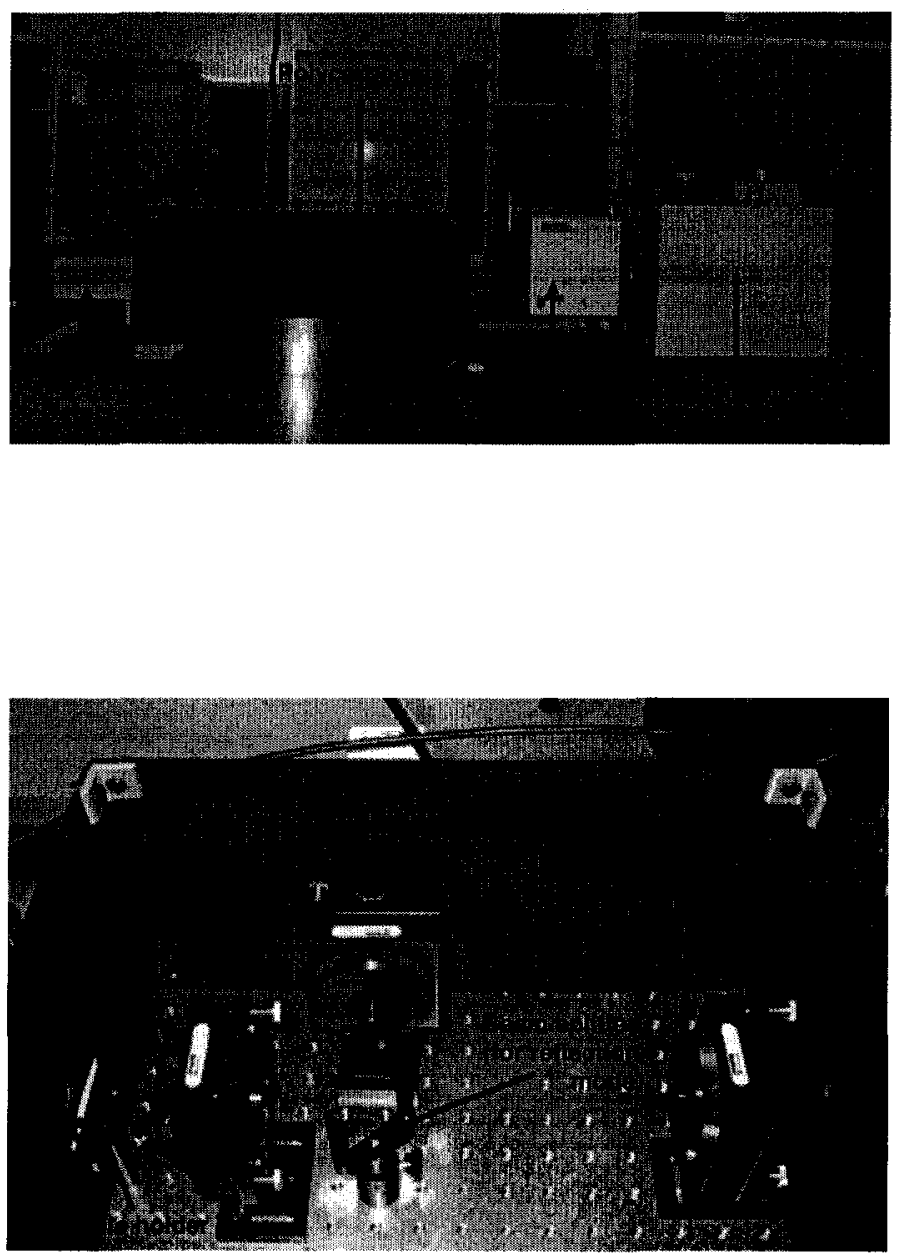

Figure 3.4: (a) spectrophotometer used for reflectometry and (b) polycarbonate box: monochromator light passes though lens 1 , lens 2 , and then through the sample holder to the detector. 


\section{Optical model}

Several optical models were tested for the refractive index $n$, extinction coefficient $k$ and the structure of anodic alumina films formed between 30 and $90 \mathrm{~V}$ in $5 \%$ phosphoric acid. The model which best fit the measured data is shown in Fig. 3.5. This model consisted of a glass substrate and a dielectric film on top of a dielectric/aluminium layer mixture. The optical parameters of the dielectric layer were modeled using a Cauchy approximation and are given below.

$$
\begin{aligned}
n & =a_{0}+\frac{a_{1}}{\lambda^{2}}+\frac{a_{2}}{\lambda^{4}} \\
k & =b_{0}+\frac{b_{1}}{\lambda^{2}}+\frac{b_{2}}{\lambda^{4}} \\
N & =n-j k
\end{aligned}
$$

$n$ and $k$ are the refractive index and extinction coefficients respectively, $N$ is the complex index of refraction and $\lambda$ is the wavelength. The variables $a_{0}, a_{1}, a_{2}, b_{0}, b_{1}$ and $b_{2}$ are the Cauchy parameters.

The optical constants of the metal/dielectric mixture were modeled using the Bruggemann effective medium approximation (EMA). When modeling the optical parameters of a mixture, an EMA can be used to treat the mixture as one homogeneous material. EMAs are thus valid only when the components of the mixture are small enough that the incident light [21] can not distinguish the components as being separate. As a rule of thumb, if the scale of the mixture is less than $10 \%$ the wavelength of the incident light, then EMAs considered to be valid. The Bruggemann EMA, given below, is thought to be the most accurate. 


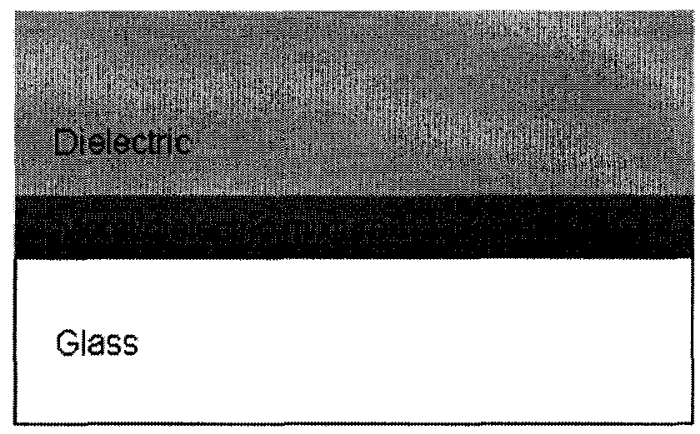

Figure 3.5: Optical model of porous alumina films

$$
f_{a} \frac{N_{a}^{2}-N_{e f f}^{2}}{N_{a}^{2}-N_{e f f}^{2}}+f_{b} \frac{N_{b}^{2}-N_{e f f}^{2}}{N_{b}^{2}-N_{e f f}^{2}}=0
$$

$N_{a}, N_{b}$ and $N_{\text {eff }}$ are the complex index of refraction of the component and effective media respectively. $f_{a}$ and $f_{b}$ are the volume fractions of the component media $[21]$.

The effective complex index of refraction $N_{\text {eff }}$ for the dielectric/metal layer mixture was obtained by substituting the Cauchy approximation into $N_{a}$ and the complex refractive index of aluminium into $N_{b}$. The optical data used for aluminium was found in [27]. The volume fractions $f_{a}$ and $f_{b}$ were treated as fitting parameters in 
the LM algorithm.

The refractive index of the glass substrate was determined using the Fresnel transmission coefficient at normal incidence. $T$ is the ratio of transmitted to incident power and $n_{s}$ is the refractive index of the corning glass. Equation 3.4 shows the Fresnel transmission coefficient for a thick transparent substrate. The first term represents the air to substrate interface and the second term represents the substrate to air interface.

$$
T=\left[\frac{2 n_{0}}{n_{s}+n_{0}}\right]^{2}\left[\frac{2 n_{s}}{n_{s}+n_{0}}\right]^{2}
$$

With equations $3.2,3.3$ and 3.4 the optical transfer functions of the dielectric and metal/dielectric mixture layers in Fig. 3.5 were obtained. The elements of the optical transfer functions were then used to calculate the transmittance of the entire multi-layer system. The optical transfer function and the transmittance of the entire system are shown in equations 3.5-3.9. A complete derivation can be found in [28]. 


$$
\begin{aligned}
& M_{o x}=\left[\begin{array}{cc}
\cos k_{o x} & \frac{j s i n k_{o x}}{Y_{o x}} \\
Y_{o x} \sin k_{o x} & \cos k_{o x}
\end{array}\right] \\
& M_{m}=\left[\begin{array}{cc}
\cos k_{m} & \frac{j \sin k_{m}}{Y_{m}} \\
Y_{m} \sin k_{m} & \cos k_{0} h
\end{array}\right] \\
& M=M_{o x} M_{m} \\
& Y_{o x}=\sqrt{\frac{\epsilon_{0}}{\mu_{0}}} N_{o x} \quad Y_{m}=\sqrt{\frac{\epsilon_{0}}{\mu_{0}}} N_{m} \\
& Y_{0}=\sqrt{\frac{\epsilon_{0}}{\mu_{0}}} \quad Y_{s}=\sqrt{\frac{\epsilon_{0}}{\mu_{0}}} n_{s} \\
& k_{o x}=\frac{2 \pi}{\lambda} N_{o x} d_{o x} \quad k_{m}=\frac{2 \pi}{\lambda} N_{m} d_{m} \\
& T=\left|\frac{2 Y_{0}}{Y_{0} M_{11}+Y_{0} Y_{s} M_{12}+M_{21}+Y_{s} M_{22}}\left[\frac{2 n_{s}}{n_{s}+1}\right]\right|^{2} \times S
\end{aligned}
$$

$M_{o x}, M_{m}, N_{o x}$ and $N_{m}$ are the optical transfer functions and complex refractive indices of the oxide and mixed layers respectively. $n_{0}$ and $n_{s}$ are the refractive indices of air and the corning glass respectively. Equation 3.9 is the total power transmitted through the multi-layer dielectric film, including the reflection of light off the back side of the glass substrate [21]. The parameter $S$ is a scattering coefficient to model the effects of Mie scattering in the alumina films inherited from the grain structure of the parent aluminium films.

\subsection{Results}

After electron beam evaporation on to the glass substrates, the aluminium films were cleaned in acetone and iso-propanol. The aluminium films were shiny and 
mirror like, but were slightly foggy. FE-SEM images revealed the grain structure of the aluminium films to be on the order of half a micron. The fogginess could be explained as Mie scattering, which is very weakly wavelength dependant and thus results in a hazy white appearance [28].

When a voltage between 30 and $90 \mathrm{~V}$ was applied to the apparatus, small bubbles would appear at the cathode, presumably hydrogen gas. Voltages greater than $100 \mathrm{~V}$ resulted in electrolytic and or dielectric breakdown, characterized by vigorous evolution of gas from both electrodes. When the reaction was complete the aluminium film turned transparent. Due to an increased anodization rate at the edges of the polycarbonate tube a ring of metal often remained in the center of the alumina film when voltages greater than $50 \mathrm{~V}$ were applied to the apparatus. A Scanning Electron Microscope (SEM) image of alumina anodized at $70 \mathrm{~V}$ is shown in Fig. 3.6. The image confirms the presence of a highly ordered porous film.

The total reaction time was measured using a stopwatch. For each applied voltage the etch rate was calculated by dividing the known thickness of the parent aluminium films $(500 \mathrm{~nm})$ by the reaction time. Figure 3.7 plots the inverse of the etch rate vs applied voltage.

The results show that in the range of 30 to $90 \mathrm{~V}$ the etch rate is approximately inversely proportional to the applied voltage, indicated by the linear nature of 3.7.

\subsubsection{Anodic Current}

The current behavior for a 30V anodization is shown in Fig. 3.8. The initial spike and exponential fall in current correspond to the formation of a barrier-type layer. The subsequent plateau in current correspond to the initiation and steady state formation 


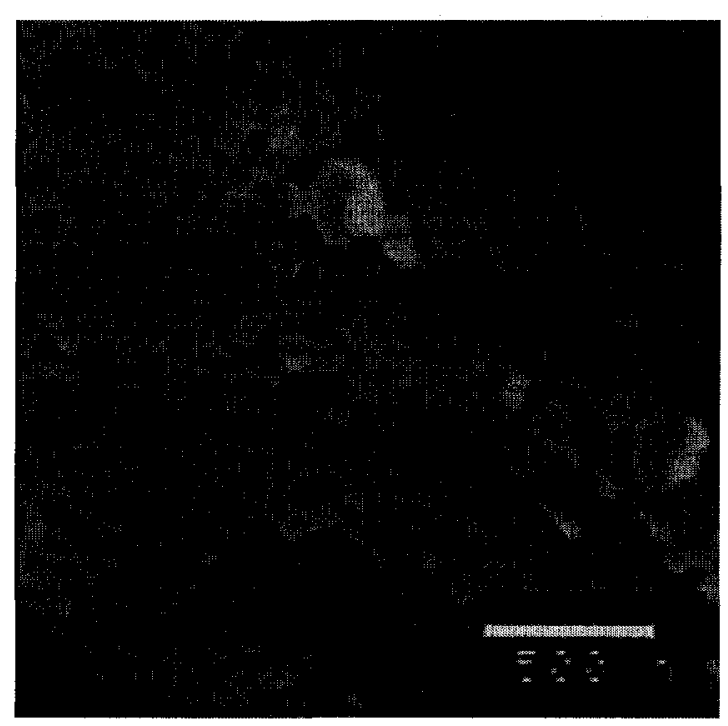

Figure 3.6: Alumina films anodized at $70 \mathrm{~V}$.

of a porous oxide $[2,18]$. These results were in agreement with observations recorded in the literature $[2,18]$.

\subsubsection{Optical characterization}

Before attempting to measure the optical parameters of the alumina films the transparency of the glass substrate was measured using the spectrophotometer described in section 3.1.3. The measured data was fit to Eq. 3.4 using the LM algorithm described in section A. A Cauchy approximation similar to Eq. 3.2 was used to model the refractive index. The extinction coefficient was assumed to be zero. The results are shown in Fig. 3.9(a) and (b). Figure 3.9(a) shows the measured data compared to the modeled data. Figure 3.9(b) shows the refractive index. The results confirm that the refractive index of the substrate varies between 1.6 and 1.5 , typical for glass. 


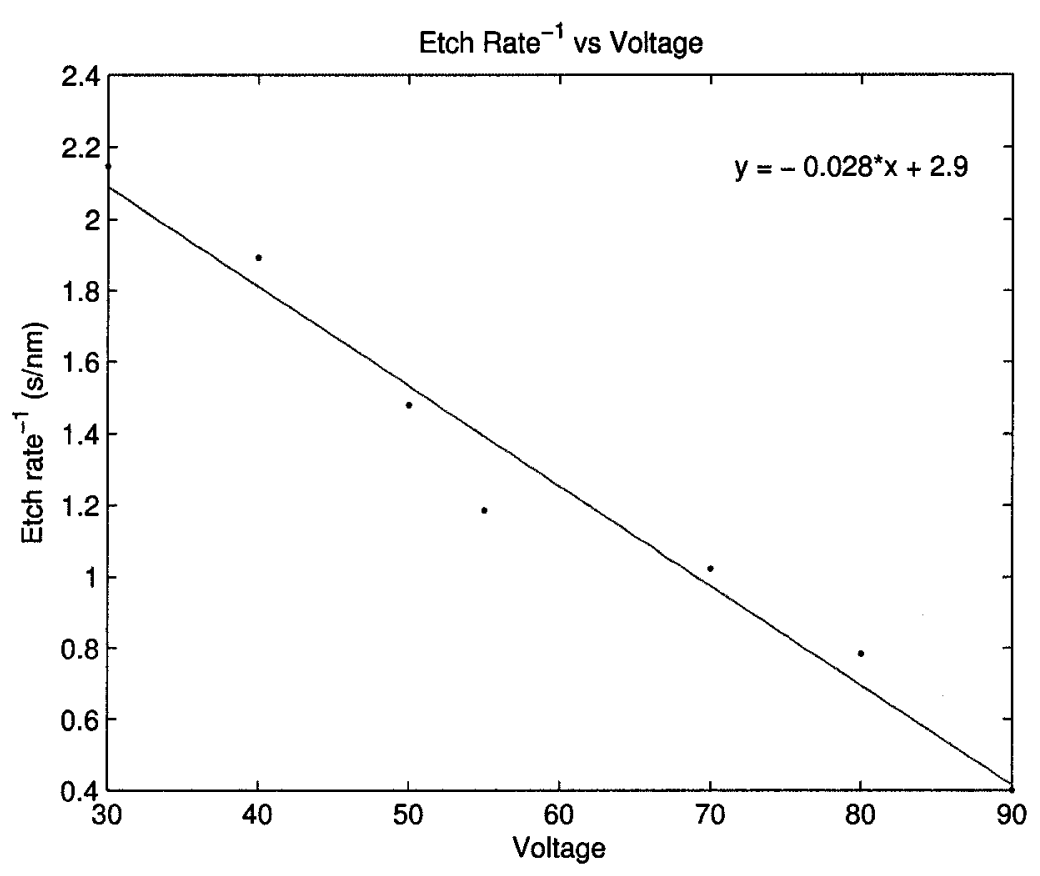

Figure 3.7: Inverse of etch rate vs applied voltage for $30-90 \mathrm{~V}$

The Cauchy parameters are shown in equation 3.10. $n$ is the refractive index and $\lambda$ is the wavelength measured in $\mu \mathrm{m}$.

$$
n=1.52+\frac{0.011}{\lambda^{2}}
$$

Several optical models were used to fit the transparency data from the alumina films. The simplest model consisted of a thick oxide layer on a glass substrate. The next simplest model consisted of a thick oxide layer on top of a very thin layer aluminium followed by the glass substrate. For both models the optical parameters of the oxide layer were approximated with Cauchy parameters using equation 3.2. For the oxide/metal/glass model the optical data for aluminium was taken from [27]. 


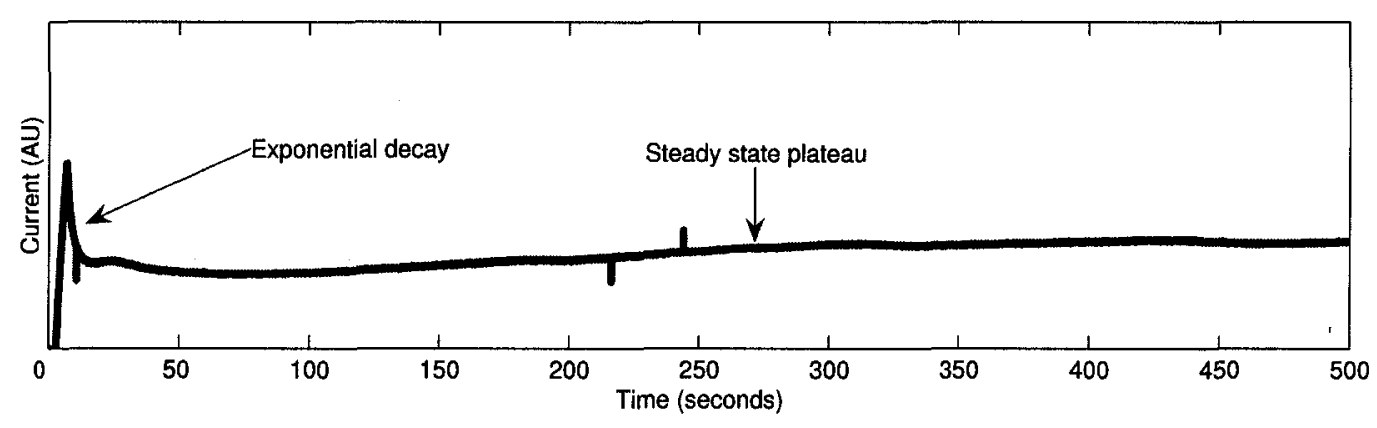

Figure 3.8: Anodic current for a 30V anodization.

The Cauchy parameters and the optical data for aluminium were substituted into equations 3.5-3.9 and the LM algorithm was used to fit each model to measured transparency data from alumina films anodized between 30 and $90 \mathrm{~V}$. In the case of the oxide/glass model the nonlinear regression parameters were the oxide thickness, the Cauchy parameters and the scattering coefficient $S$. The oxide/metal/glass model included the same parameters plus the thickness of the thin metal layer.

Both of these models were inadequate at predicting the behavior of the transparency data for the alumina films. Figures 3.10(a) and (b) show the measured and modeled data for the transparency of an alumina films anodized at 50V. Figure 3.10(a) shows the results from oxide/glass model and Fig. 3.10(b) shows the results from the oxide/metal/glass model. In both cases, the fit is close, however the 
Figure A

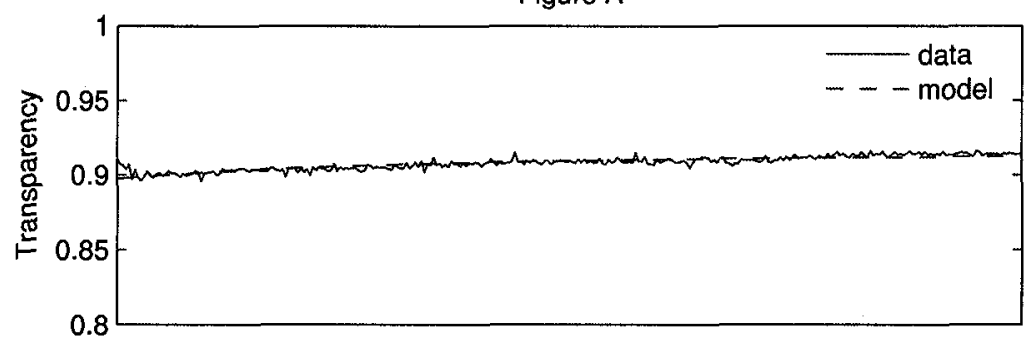

Figure B

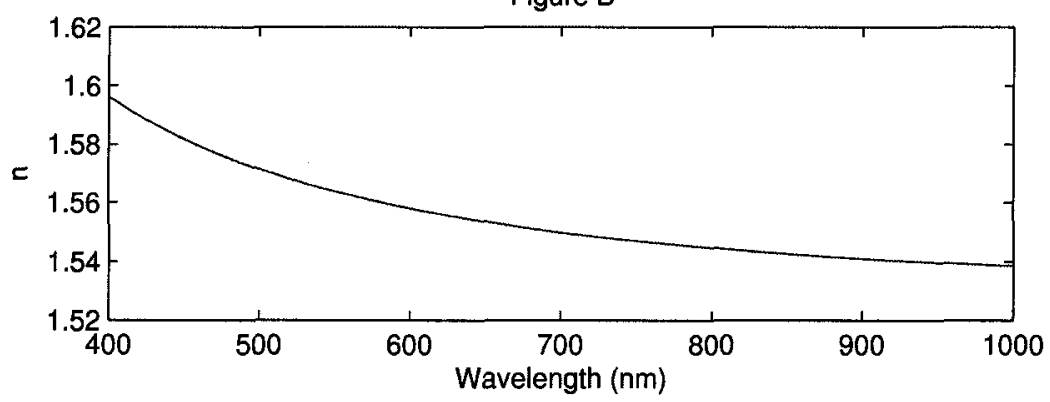

Figure 3.9: Refractive index of glass substrate

peaks do not entirely overlap, and a few are slightly out of phase.

A much better fit to the data was attained using the model discussed in section 3.1.3, shown in Fig. 3.5. This model consisted of a thick oxide layer on top of a mixture of oxide and metal and then a glass substrate. The regression parameters were the Cauchy parameters, the thickness of the oxide, the thickness of the oxide/metal mixture and the volume ratio of oxide to metal. Once again, the optical data for aluminium was taken from [27]. Eq. 3.3 was used to calculate the effective index of the metal/oxide mixture. The LM algorithm was used to fit Eq. 3.5-3.9 to the measured data. The measured and modeled data for alumina films anodized at 50 volts is shown in Fig. 3.11. The model and data are nearly identical. A similar fit was attained for films anodized at the following voltages: $30,40,55,70,80$ and 
Figure A

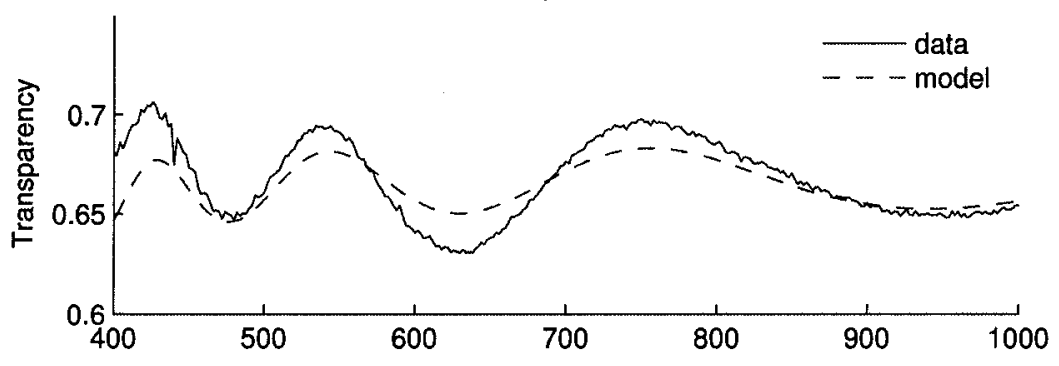

Figure B

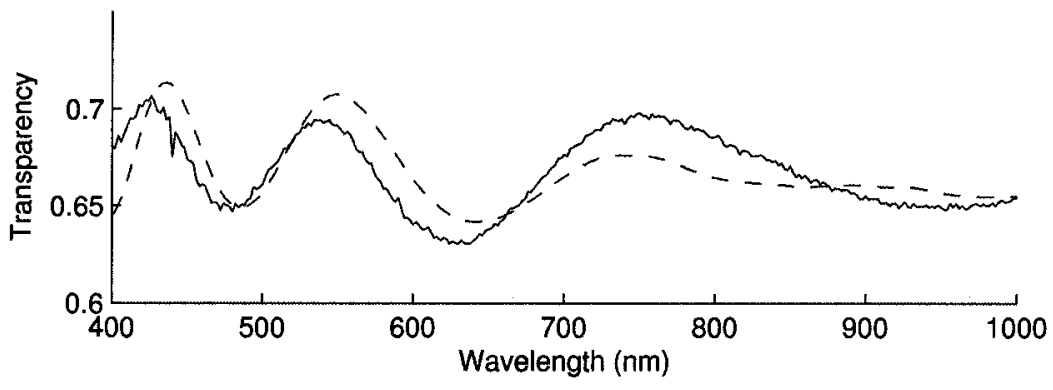

Figure 3.10: Simple optical models for alumina films. Figure (a) is the result of the simplest model, pure oxide on a glass substrate. Figure (b) is the result of the next simplest model, pure oxide on a thin layer of pure metal on a glass substrate.

$90 \mathrm{~V}$. The values of refractive index for the all oxide layers measured are shown in Fig. 3.12. The extinction coefficient was found to be very small and had minimal effect on the fit of the model to the data. For this reason the extinction coefficient of the oxide layer was set to zero for the LM regression.

Figure 3.12 shows that the refractive index of the anodic films increases rapidly with applied voltage and then tapers off at around $70 \mathrm{~V}$. The reasons for this will be discussed in the next section.

The scattering coefficient and film thickness obtained through the LM regression are shown in Fig. 3.13(a) and (b) respectively. The values of film thickness obtained 


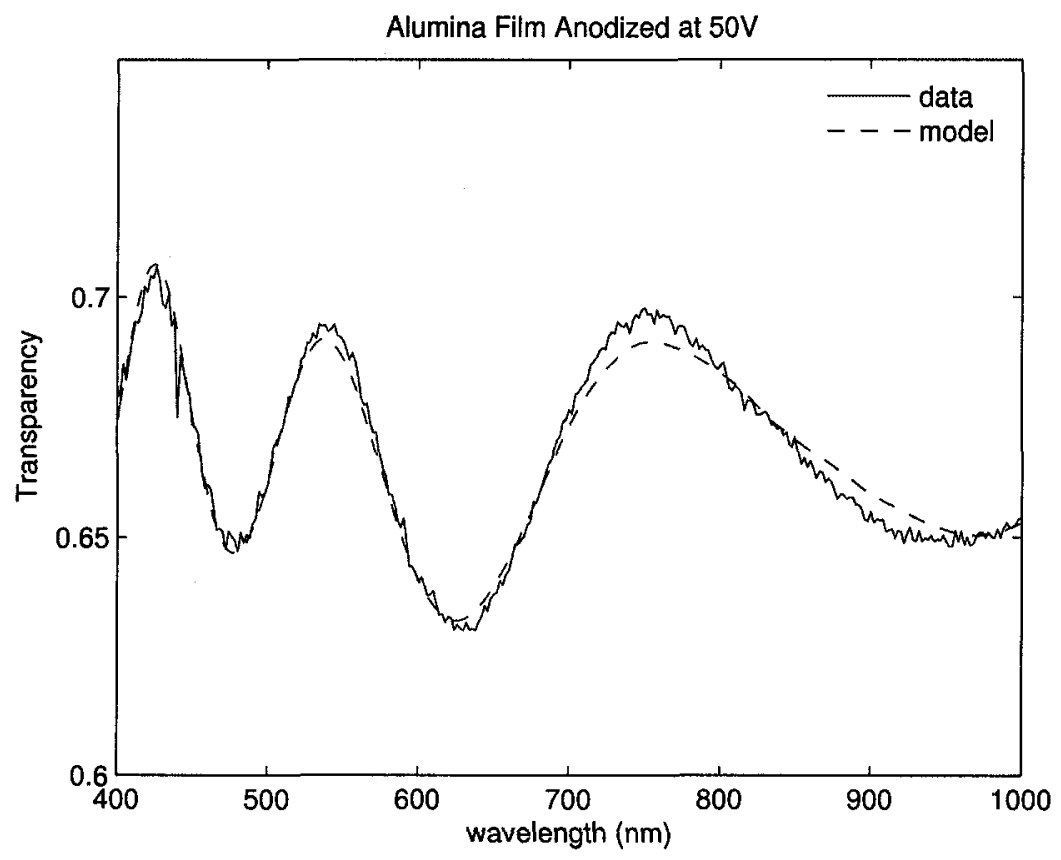

Figure 3.11: Measured and modeled data for alumina film anodized at 50V.

from the optical data show close agreement with measurements taken with the Tencor P1 long scan profiler. The data shows that the thickness of the oxide film resulting from $500 \mathrm{~nm}$ of $\mathrm{Al}$ is approximately linear in the range of voltages explored. Similar results were reported in [20]. The scattering coefficient also shows variability with voltage. This is most likely due to the change in refractive index and film thickness with voltage. Mie scattering is known to be wavelength independent and related to the number and refractive index of the scatterers. 


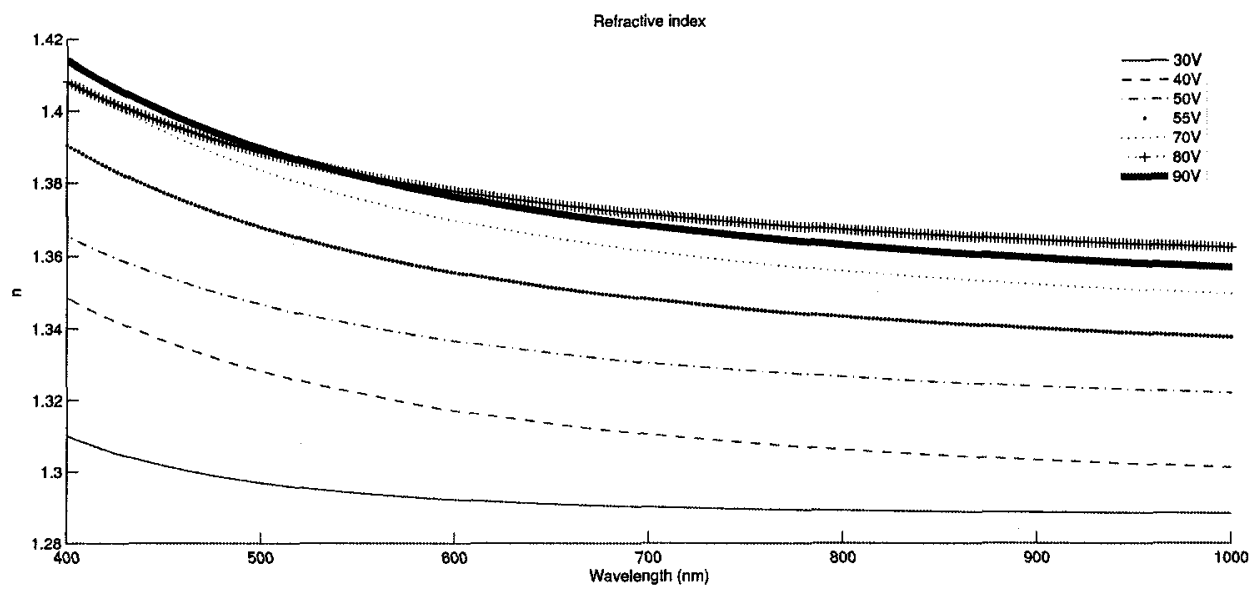

Figure 3.12: Refractive index for alumina films anodized between 30 and $90 \mathrm{~V}$.

\subsection{Discussion}

The results presented in this chapter have shown that highly transparent and well ordered nanostructured alumina films can be produced by anodizing electron beam evaporated aluminium in $5 \%$ phosphoric acid solution. It was shown that the etch rate and the final thickness of the oxide can be controlled by the anodizing voltage. Measurement of oxide thickness using optical data and the Tencor P1 long scan profiler are in close agreement. Scattering of light in the alumina film appears to be inherited from the grain structure of the parent aluminium film. The scattering coefficient is found to be dependant on applied voltage. This dependance is thought to arise from changes in film thickness and refractive index.

An optical model is presented which accurately fits transparency data measured with a spectrophotometer. The optical model consists of a layer of pure oxide, a thin mixture of oxide and metal and a glass substrate. The success of this model suggests 

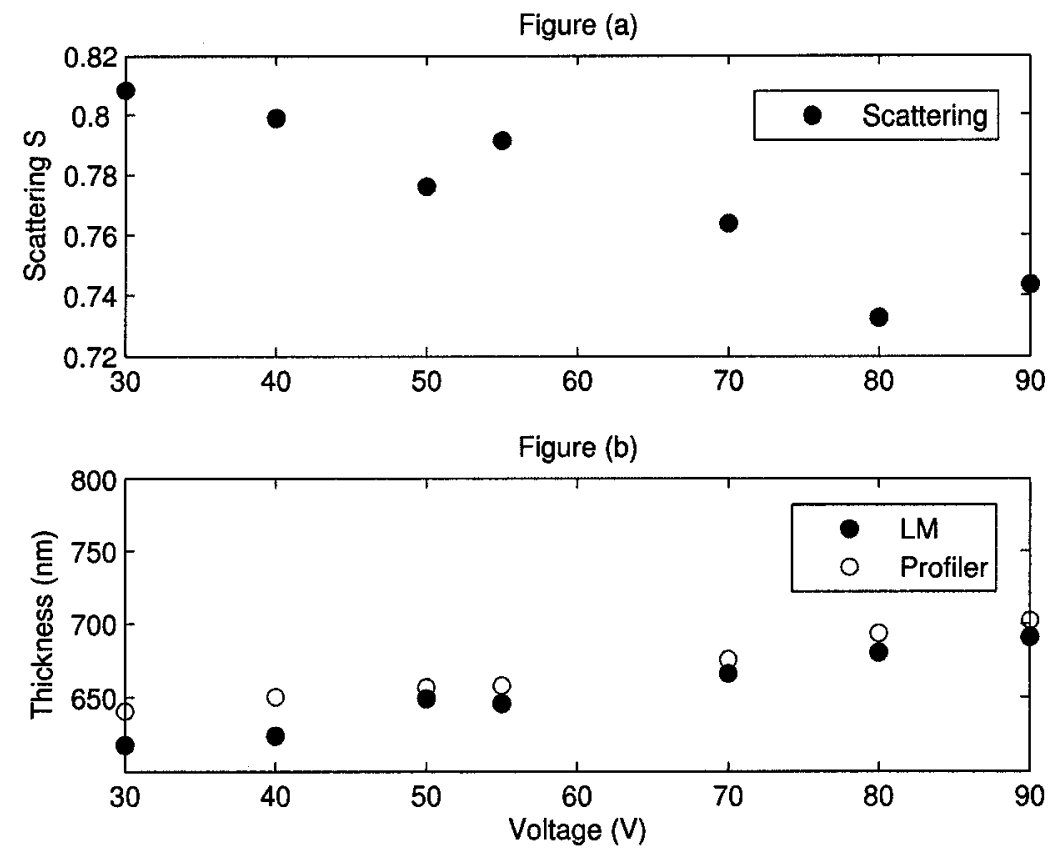

Figure 3.13: Film thickness resulting from $500 \mathrm{~nm}$ of $\mathrm{Al}$ and scattering coefficient for films anodized between 30 and $90 \mathrm{~V}$.

that as the anodization reaches completion the parent aluminium is nearly all consumed. Small pockets (less than one tenth the wavelength of light) are electrically isolated from the anodic current and are therefore left un-oxidized. The result is a very thin layer directly above the glass substrate where small amounts of residual metal remain even after the reaction has been completed.

It has been established that both the pore radius and pore spacings of anodic alumina are linearly proportional to the applied voltage $[9,20]$. The porosity of the anodic alumina is given by the ratio of the pore volume to the entire volume of oxide. Figure 3.14 illustrates a simple 2D model for determining the relationship between porosity and voltage. 


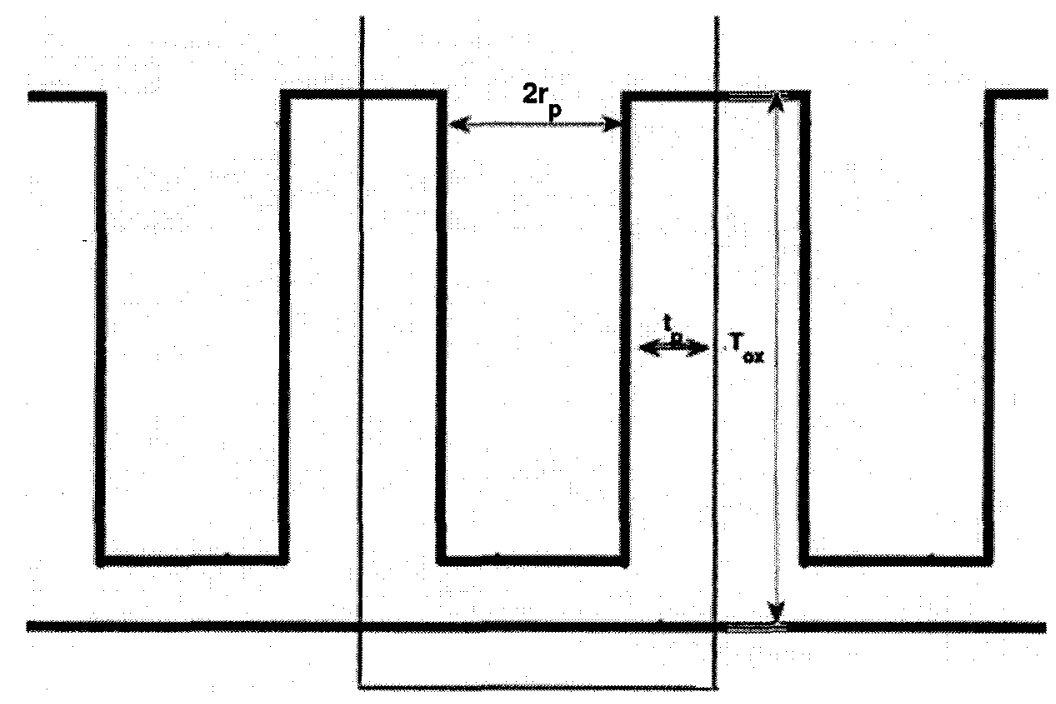

Figure 3.14: Simple 2D model for porous alumina.

In this model, the unit cell of the anodic alumina is the region encapsulated by the thin black line. The pore radius is $r_{p}$ and the inter-pore spacings are $t_{p}$. The barrier layer can be ignored because it is much small than the total thickness $T_{o x}$ of the film. Based on these assumptions, a simple model for the porosity of the alumina film as a function of voltage can be established. The total pore volume for one unit cell in two dimensions neglecting the barrier layer is given by

$$
V_{p}=2 r_{p} T_{o x}
$$

The total volume of one unit cell is given by 


$$
V_{c}=2\left(r_{p}+t_{p}\right) T_{o x}
$$

where $V_{p}$ and $V_{c}$ are the pore and unit cell volumes respectively. These two equations can be combined to obtain an expression for the porosity. Since $r_{p}$ and $t_{p}$ are both known to be proportional to the applied voltage $V$, expression for them in terms of $V$ can also be introduced $[9,20]$.

$$
\begin{aligned}
r_{p} & =\alpha V \\
t_{p} & =\beta V \\
P & =\frac{V_{p}}{V_{c}}=\frac{2 r_{p} T_{o x}}{2\left(r_{p}+t_{p}\right) T_{o x}} \\
P & =\frac{\alpha V}{\alpha V+\beta V} \\
P & =\frac{\alpha}{\alpha+\beta}
\end{aligned}
$$

Equation 3.13 suggests that the porosity of the alumina films is independent of the applied voltage. This contradicts the results presented in Fig. 3.12 that show for low anodizing voltages the refractive index increased quickly with voltage and then levels off at around $70 \mathrm{~V}$, which suggests that the porosity is also varying with voltage. The reason for this is thought to be that the microstructure of the parent aluminium film dominates the dimensions of the pores at low voltages, when the pore size is much smaller than the grain structure of the aluminium film. As the applied voltage increases, the microstructure has less effect on the pore radius and separation. 
To gain further understanding of this phenomenon alumina films anodized at 30V were imaged with FE-SEM. The images are shown in Fig. 3.15. Figure 3.15(a) demonstrates that at low magnification, the grain structure of the parent aluminium films dominates the structure of the anodic oxide. Figure 3.15(b), at a higher magnification, reveals that not only does the grain structure dominate the topography of the oxide, but that the pore size seem to be greatest in between the grains. It is likely that this uneven distribution of pores is what is causing the unexpected change in porosity, and therefore refractive index.

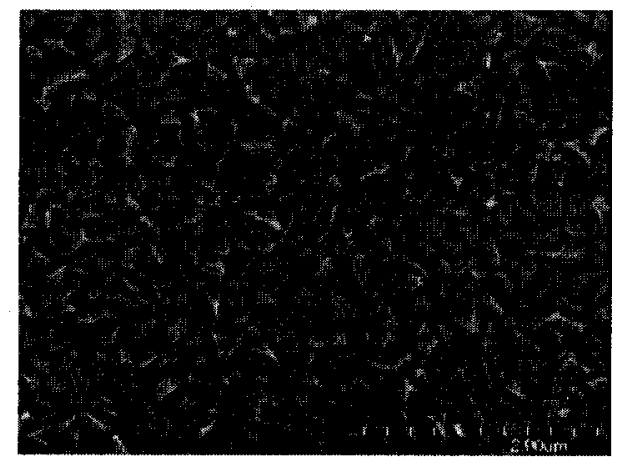

(a)

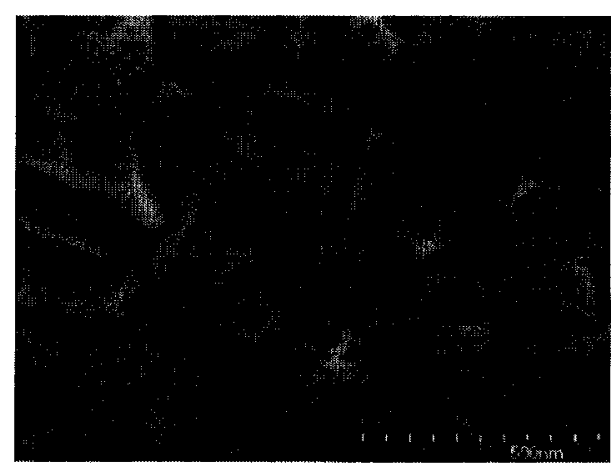

(b)

Figure 3.15: Alumina film anodized at 30V under high and low magnification.

This hypothesis is consistent with the experimental procedure outlined in the literature. To increase the degree of ordering and obtain good control over the pore size and separation researchers have typically preformed cycled depositions to inhibit grain formation and electropolished the aluminium anode before anodizing [3]. Electropolishing smooths out imperfections that otherwise would dominate the pore structure at low voltages. 


\subsection{Summary}

This chapter explored porous alumina films fabricated by anodizing electron beam evaporated aluminium films in a $5 \%$ phosphoric acid solution. The alumina films were highly ordered, adhered well to a glass substrate and were transparent. The etch rate and the final thickness of alumina after complete conversion of the aluminium anode film were found to be inversely and directly proportional to applied voltage respectively, in the range of voltages explored. An optical model was proposed and tested using transparency data and the LM algorithm. A good fit for the optical model was achieved for alumina anodized between $30-90 \mathrm{~V}$. The refractive index was shown to increase quickly for low anodizing voltages and then remained consistent at $70 \mathrm{~V}$. Contrary to equation 3.13 , this suggested that the porosity of the alumina films was varying with voltage, for low voltages. FE-SEM images confirmed that the pore size is not well controlled at low voltages, most likely because of the microstructure of the parent aluminium films. It was suggested that cycled depositions and electropolishing before anodizing might help to alleviate this problem. 


\section{Chapter 4}

\section{Nanostructured Titania thin films: Fabrication and Characterization}

One of the most active research areas for NST are dye sensitized solar cells. Early experimentation with anodically grown alumina quickly revealed that the process was not compatible with ITO coated substrates. When aluminium was deposited onto an ITO coated substrate and anodized, the ITO immediately delaminated from the glass substrate, removing the aluminium film with it. Similar experiments with titanium films resulted in the same effect. Since ITO coated substrates are necessary for DSC operation, in-situ formation of NST through the anodic oxidation of titanium was abandoned. The chemical oxidation method described in chapter 2 was found to be compatible with ITO coated substrates. Furthermore, unlike anodic films, it was also found to be compatible with heat treated polyimide and polyester substrates, opening the door to printable electronics and roll to roll processing of flexible DSCs.

In this chapter we investigate the formation and properties of NST created by 
reacting sputtered titanium films with 10 percent hydrogen peroxide $[4,5,19]$. Provided is a detailed analysis of the film structure, porosity, intrinsic stress and surface area. FE-SEM cross-sections are used to analyze the physical structure of the NST before and after annealing. Fourier Transform Infrared Spectroscopy (FT-IR) is used to gather information about the composition of the as-oxidized and annealed films. Pre-patterning the parent Titanium films with a lift off technique has been shown to inhibit cracking of NST during oxidation $[4,5]$. Here the effect of tensile and compressive stresses in the initial Titanium films are measured and used to create a process where crack free NST can be produced without the use of pre-patterning. Detailed analysis of the surface area by the BET method is presented for the first time. The effect of deposition pressure on surface area is also explored. The specific surface area of the NST film, measured for the first time, is found to be $535 \mathrm{~m}^{2} / \mathrm{g}$, among the highest reported for nanostructured Titania [22, 29]. Optical characterization of the NST films confirm that they are highly transparent, a property which is critical for its potential use as a photo-catalyst as well in dye-sensitized solar cells. To demonstrate the potential use of the NST in dye-sensitized solar cells annealed films are dyed in $1 \times 10^{-4} \mathrm{M}$ eosin-y ethanol. The dyed films exhibit substantial absorption of light in the visible spectrum.

\subsection{Experimental procedure}

\subsubsection{NST preparation}

Titanium films were planar RF sputtered at 2.5, 8 and 25 mTorr on silicon and glass at $300 \mathrm{~W}$ for $45 \mathrm{~min}$, following a 30 minute pre-sputter of the titanium target. The 
resulting sputtered Titanium layers ranged from 0.7 to $0.8 \mu \mathrm{m}$ thick, as measured using SEM cross-sections. A WYKO MHT-3 optical profiler was used to measure stress in the deposited titanium films. The samples were cleaned in acetone and isopropanol, and then dried in a nitrogen stream. Titanium films sputtered at 2.5, 8 and 25 mTorr were oxidized for 45 minutes. Following the oxidation the samples surface area was determined. After annealing the surface area analysis was repeated. For transparency measurements Titanium sputtered on glass microscope slides at 25 mtorr was oxidized in $10 \% \mathrm{H}_{2} \mathrm{O}_{2}$. When the samples transformed from opaque to nearly transparent, indicating the near complete consumption of the metal layer, they were removed from solution and annealed at $300^{\circ} \mathrm{C}$ for 10 hours. Due to equipment limitations 25 mTorr was the highest process pressure that could be used.

\subsubsection{Density calculation}

The mass of the titania film is required for specific surface area measurements, which are in units of $m^{2} / g$. Since obtaining a direct measure of mass by weighing the films was difficult, a separate set of experiments were devised to determine the density the titania films.

Titanium films were sputtered and then oxidized in exactly the same conditions as described above. The total mass of titanium before and after the oxidation was calculated using:

$$
M=t A \rho
$$

where $\rho$ is the density of bulk titanium, $t$ is the thickness of the titanium film and $A$ is the area of the silicon wafer. The parameter $t$ was determined by SEM cross 
section, see Fig. 4.1(a). While one would expect $\rho$ for sputtered Titanium to be to somewhat smaller than bulk titanium, for these calculations the bulk value was used because it resulted in a lower limit for SSA.

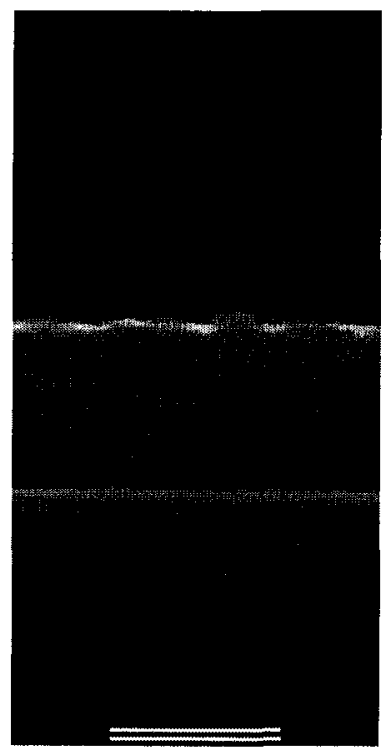

(a)

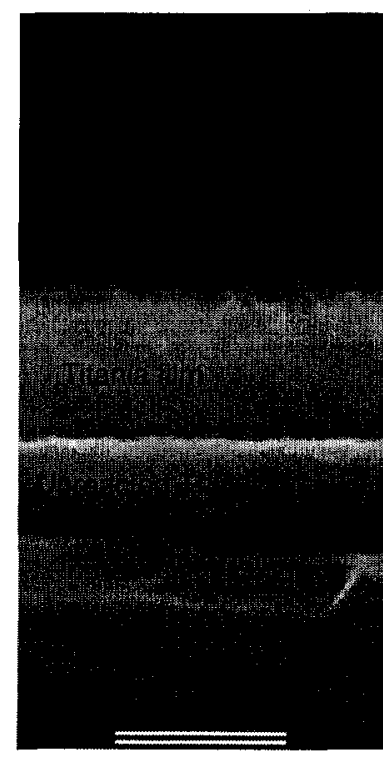

(b)

Figure 4.1: SEM images of titanium films (a) before and (b) after oxidation

Once the mass of the original and remaining Titanium layers was known, the oxide density could be calculated using:

$$
\rho_{o x}=\frac{M M_{o x}}{V_{o x} M M_{T i}}\left[M_{i}-M_{r}-M_{a q}\right]
$$

This equation makes the assumption that the composition of the oxide film is mostly $\mathrm{TiO}_{2} . M M_{o x}$ is the molar mass of titanium dioxide, $M M_{T i}$ is the molar mass of titanium, $M_{i}$ and $M_{r}$ are the masses of the original and remaining titanium films 
respectively. $V_{o x}$ is the volume of the oxide as determined from the SEM crosssections and the known area of the substrate, see Fig. 4.1(b). $M_{a q}$ is the mass of aqueous titanium as determined from inductively coupled plasma (ICP) mass spectroscopy of the remaining $\mathrm{H}_{2} \mathrm{O}_{2}$ solution after the oxidation process. The value of $\rho_{o x}$ calculated for a specific set of growing conditions was assumed to be constant when these growing conditions were repeated.

\subsubsection{Optical characterization}

The transparency of annealed and dyed films on glass substrates was measured using the spectrophotometer described in section 3.1.3. The films were dyed using a $1 \times 10^{-4}$ M eosin-y (Aldrich) ethanol solution. The NST and glass substrates were heated to $80^{\circ} \mathrm{C}$ for 20 minutes to remove adsorbed water before being placed in the dye solution. After 24 hours, the samples were removed and rinsed in pure ethanol. The transparency of the samples was then measured again.

\subsection{Results}

\subsubsection{Film appearance and structure}

After removal from the $\mathrm{H}_{2} \mathrm{O}_{2}$ solution, the titanium films that were deposited on silicon substrates had turned a dark brown or golden color. The NST film was fragile easily scratched if mishandled. After annealing, the samples turned either red or green in color and became resistant to scratching. For titanium films that were deposited on glass, upon removal from the $\mathrm{H}_{2} \mathrm{O}_{2}$ the transparency of the films indicated that the metal layer was completely consumed. Oxidation/etching of the 
titanium layer occurred faster along the edges of the glass slide, resulting in bare glass along the edges. After annealing the films turned nearly $90 \%$ transparent. Upon FESEM examination of films on both silicon and glass, a highly porous honeycomb like structure could be seen (Fig. 4.2), similar to that reported in $[4,5,19]$.

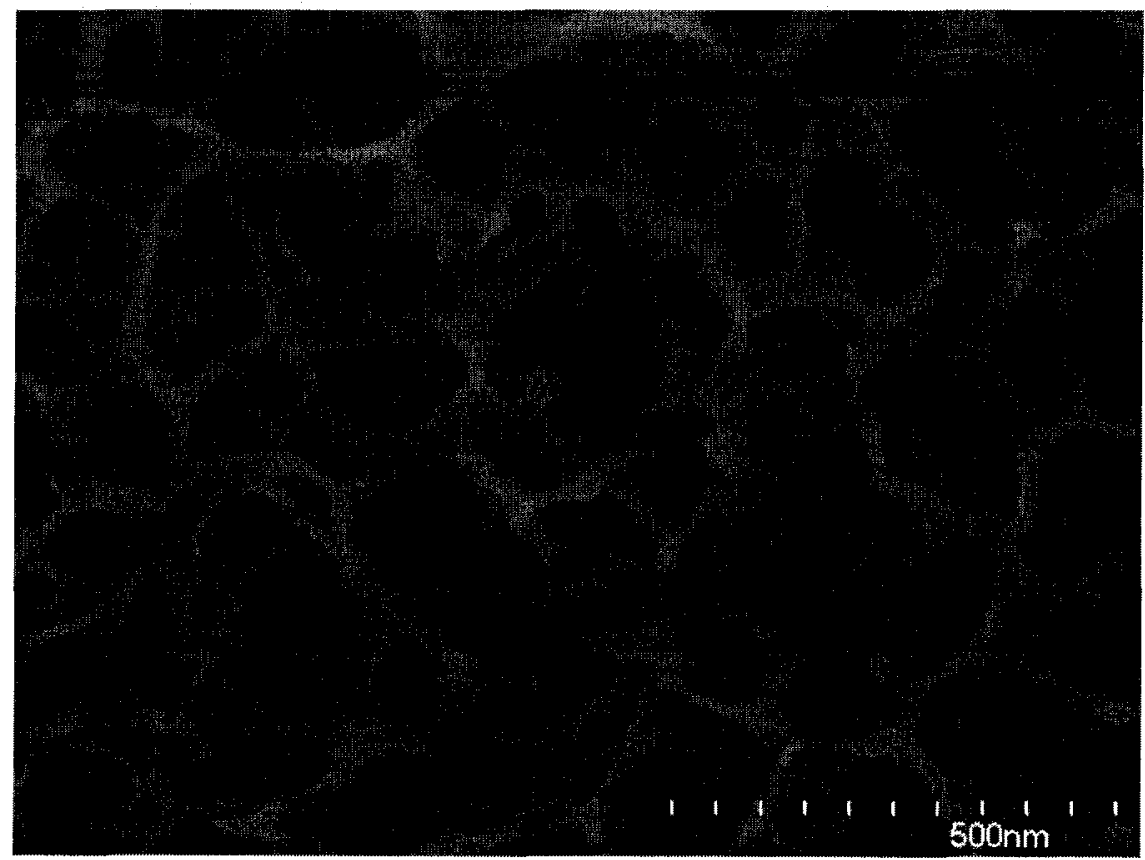

Figure 4.2: Top surface of NST derived from Titanium film deposited at $25 \mathrm{mTorr}$ and oxidized for 45 minutes in $\mathrm{H}_{2} \mathrm{O}_{2}$.

FE-SEM cross sections show two distinct layers; a highly porous layer and an intermediate layer. Figure 4.3 shows an FE-SEM cross section of NST obtained by oxidizing a Titanium film sputtered at 25 mTorr for 45 minutes. The two distinct layers are clearly visible, as well as a metal layer underneath. The existence of the 


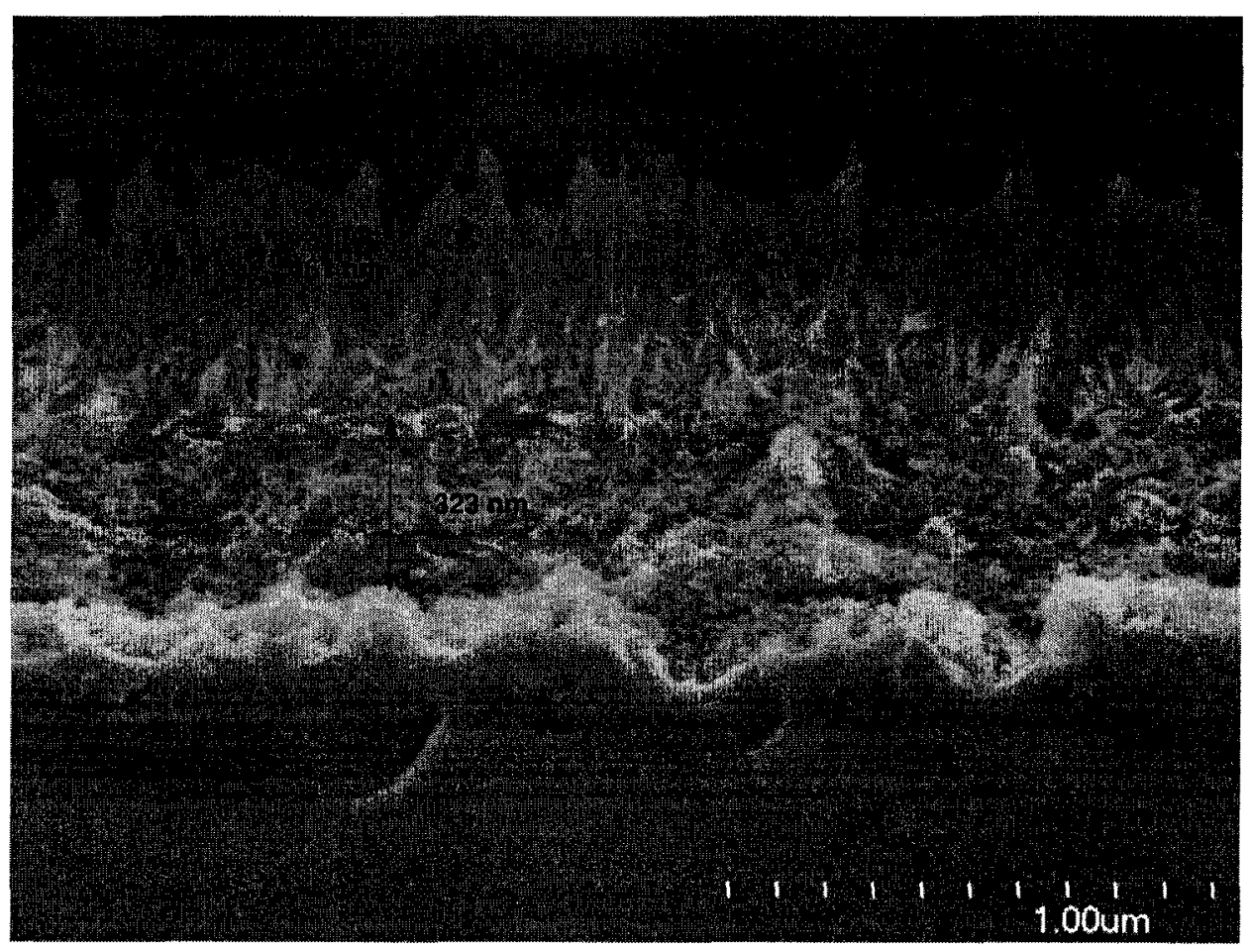

Figure 4.3: FE-SEM cross-section of NST showing the porous layer on top, an intermediary titania water complex layer and the remaining metal film.

intermediate layer between the metal and the porous oxide has not been previously reported $[4,5,19]$. In previous works it has been suggested that a peroxi-titania gel may be involved as an intermediate step in the formation of NST $[4,5,19]$. To support this, Zuruzi and others have referred to publications where a titania gel was produced by reacting titanium sheets or biomedical implants with low concentrations of hydrogen peroxide [30]. It is our contention that this intermediate layer is in fact a hydrated titania layer, (either a Lamellar clay or a titania gel) which forms at the titanium surface.

FT-IR was used to probe for more evidence of a hydrated layer. FT-IR can be 


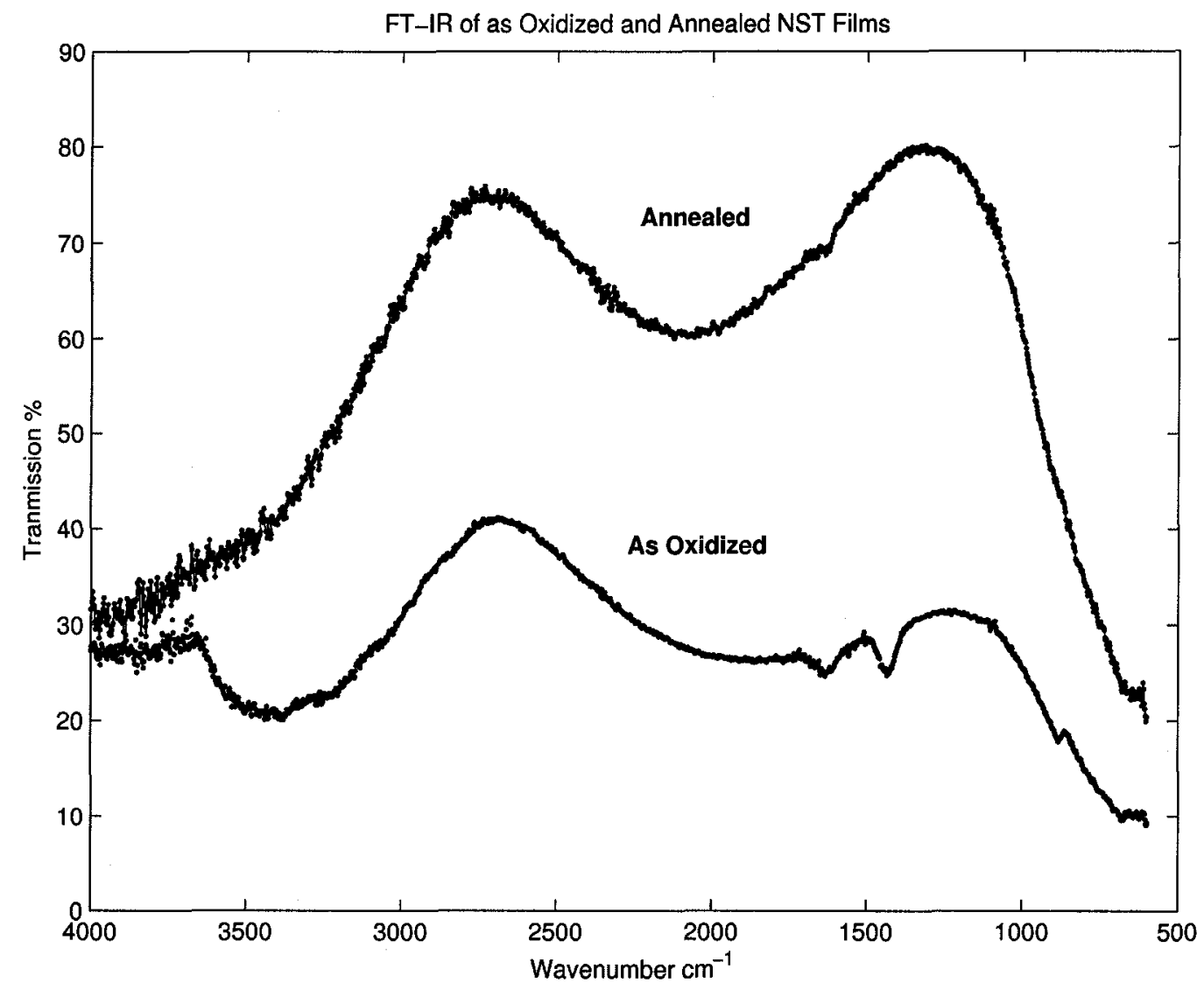

Figure 4.4: FT-IR measurements indicating $\mathrm{OH}$ bond stretching.

used to analyze the rotational and vibrational states of molecular bonds. It can be used to detect the presence of water in the NST film by detecting OH bond resonances.

To detect the presence of a hydrated titania layer as oxidized NST was removed from the beaker containing $\mathrm{H}_{2} \mathrm{O}_{2}$ and air dried for 10 minutes. Following that the NST was dried in an air stream at $80^{\circ} \mathrm{C}$ for 5 minutes so that any water remaining on the surface would evaporate before being placed in the FT-IR. The same sample of 


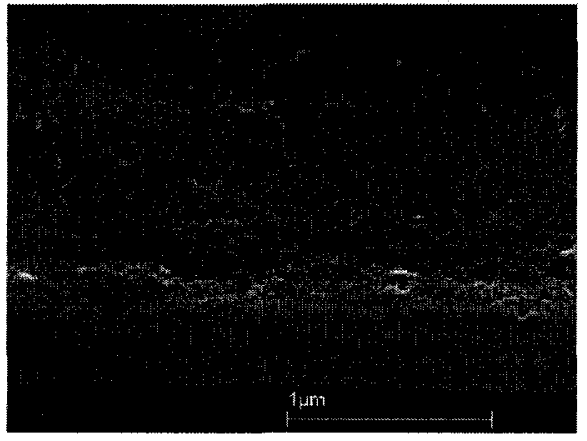

(a)

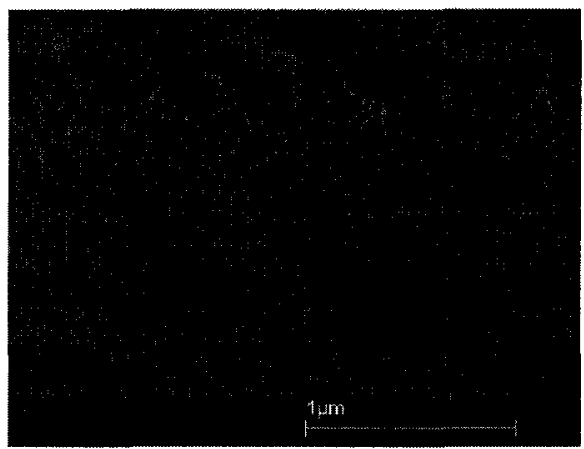

(b)

Figure 4.5: Mechanically polished cross-sections of (a) as oxidized and (b) annealed NST. The intermediary layer is more compact after annealing

NST was then annealed in a dry atmosphere at $250^{\circ} \mathrm{C}$ for 3 hours, soaked in water at $90^{\circ} \mathrm{C}$ for 24 hours. The sample was then air dried for 10 minutes, blow dried exactly like the as-oxidized film and then placed in the FT-IR for analysis. The intention of this process was to eliminate the possibility of mistaking liquid water trapped in the porous film for water present in a hydrated titania layer.

The plot labeled as-oxidized, as shown in Fig. 4.4 presents the transmission FTIR spectrum of the NST after blow drying but before annealing. The results show a broad dip in transmission at around $3500 \mathrm{~cm}^{-} 1$, which is consistent with $\mathrm{OH}$ bond stretching and a smaller dip at $1400 \mathrm{~cm}-1$ which is consistent with $\mathrm{OH}$ deformation. This type of IR spectrum suggests that water is present in the titania film. The plot labeled annealed as shown in Fig. 4.4 is of the same NST after annealing for 3 hours at $250^{\circ} \mathrm{C}$ in a dry atmosphere and then soaked in water at $90^{\circ} \mathrm{C}$ for 24 hours. The annealed film shows no trace of $\mathrm{OH}$ bonds, which indicates that any water contained 
in the as-oxidized film had disappeared upon annealing, and did not come from adsorbed or trapped water. The annealed plot shows significantly more transmission across the entire measured spectrum. This is due to residual metal remaining in the as-oxidized film transforming into oxide upon annealing.

\subsubsection{Film Stress and Cracking}

Oxidation of titanium films longer than one hour produced NST films with high crack densities, identical to that reported in $[4,5,19]$. It has been suggested that crack formation was due to shrinkage of the NST during the drying and annealing process [4]. While SEM images are provided to support that the NST actually shrinks, a quick comparison of the volume of titania produced from one mole of titanium (this can be achieved by directly comparing the molar volume of Titanium to that of $\mathrm{TiO}_{2}$ ) suggests that the titanium should expand, as opposed to shrink, upon oxidation. One possible explanation is the presence of the hydrated titania layer described above. Once removed from the solution, this layer would begin to shrink due to evaporation of incorporated water.

To gain further understanding, FE-SEM was used to image mechanically polished cross-sections of the NST film before and after annealing. Figure 4.5 shows the mechanically polished cross-section of a Titanium film deposited at 8 mTorr and subsequently oxidized in 10 percent hydrogen peroxide. Figure 4.5(a) shows the NST before annealing while Fig. 4.5(b) shows the NST after annealing. The distinct layers mentioned earlier in this section are clearly visible. The top layer is the porous oxide and the bottom layer is the proposed hydrated titania layer. The FE-SEM crosssections show the hydrated titania layer to be more compact after annealing. The 


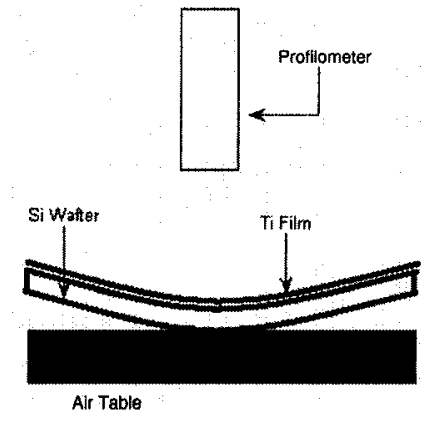

Figure 4.6: Bending of substrate due to titanium film on silicon wafer

\begin{tabular}{|l|l|}
\hline Pressure mTorr & Deflection $\mathrm{nm} / \mathrm{mm}$ \\
\hline 2.5 & -2 \\
\hline 8.0 & 18 \\
\hline 25 & 30 \\
\hline
\end{tabular}

Table 4.1: Deflection of wafers due to titanium films deposited at different pressures

reduction in volume of the hydrated titania layer was most likely caused by the loss of incorporated water, and therefore would start to occur immediately after removal of the NST from the peroxide solution. This loss in volume is thought to be the cause of the cracking in the NST. This theory is consistent with the observations made by Zuruzi et. al., where cracking was reported to start immediately upon removal of the NST from the peroxide solution. Furthermore this explains why cracking occurs only in films oxidized for greater than one hour. Longer oxidation times result in a larger amount of water incorporated into the hydrated titania layer and therefore a greater reduction in volume once it starts to dry.

To investigate crack formation from other sources, stress in the parent metal films was calculated. To control the stress in the films Titanium was sputtered at increasing gas pressures. Wafer bending of the silicon substrate was calculated using a WYKO MHT-3 optical profiler. Figure 4.6 illustrates the setup used to measure the deflection. By convention a positive deflection represents a concave surface, indicating that the film is in tension. Table 4.1 illustrates that with increasing 


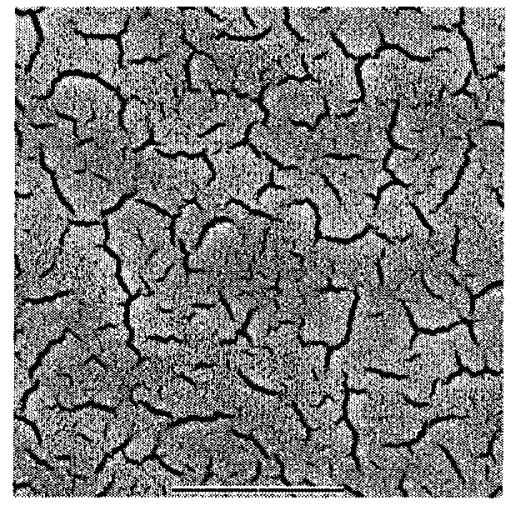

(a)

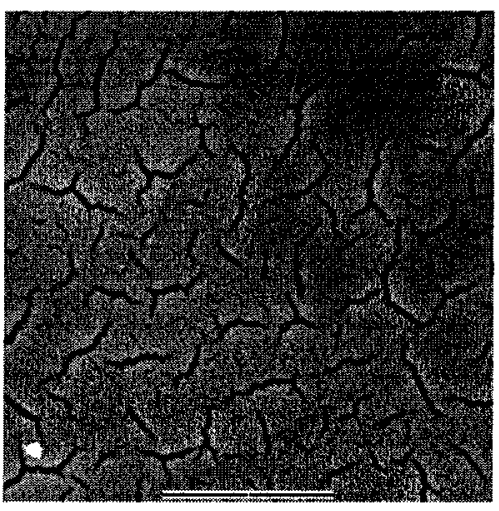

(b)

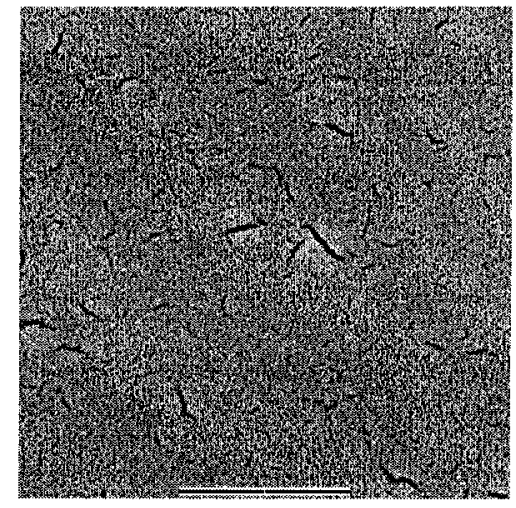

(c)

Figure 4.7: FE-SEM images of NST surface for different titanium sputtering pressures (a) 2.5 mTorr, (b) $8 \mathrm{~m}$ Torr and (c) 25 mTorr respectively. A $10 \mu \mathrm{m}$ bar is shown in each.

deposition pressure the stress in the Titanium films is increasingly tensile. Figure 4.7 shows the NST resulting from Titanium deposited at a) 2.5 mTorr, b) 8 mTorr and c) 25 mTorr. All samples were oxidized for 90 minutes. Titanium deposited at 25 mTorr produces NST with significantly less crack formation than films deposited at lower pressures. This is somewhat confusing as the Titanium film deposited under the most tension produces NST with the lowest crack density. The reasons for this are not clear, but this indicates that tensile forces due to water loss are more important in crack formation than tensile forces associates with the sputter deposition.

\subsubsection{Film Surface Area and Porosity}

Large surface areas for the as-oxidized films were measured using BET nitrogen isotherms. Figure 4.8(a)-(c) show the BET isotherms for NST oxidized from titanium films deposited at 2.5, 8 and 25 mTorr respectively. All three isotherms exhibit 
(a) 2.5 mtorr

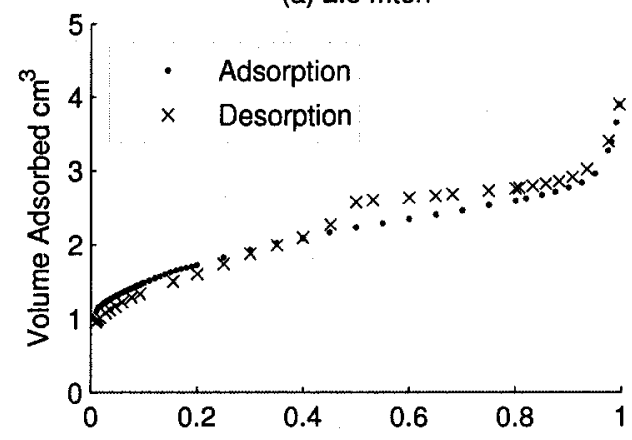

(c) 25 mtorr

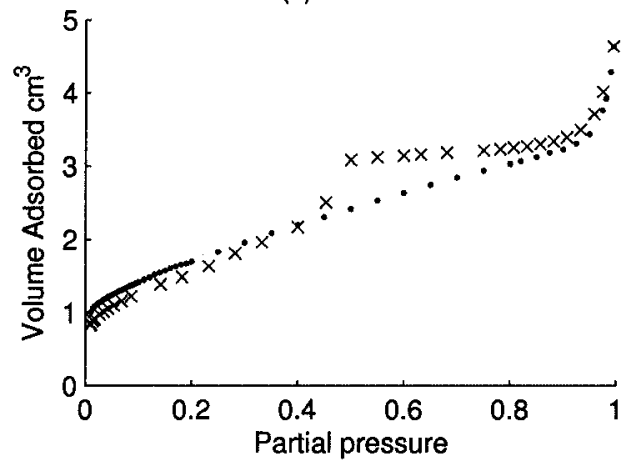

(b) 8 mtorr

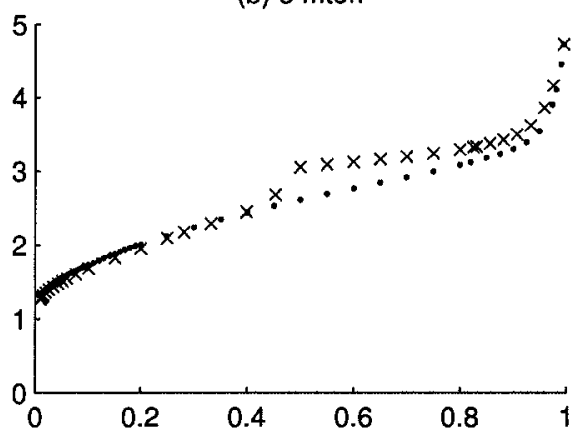

(d) 25 mtorr Annealed

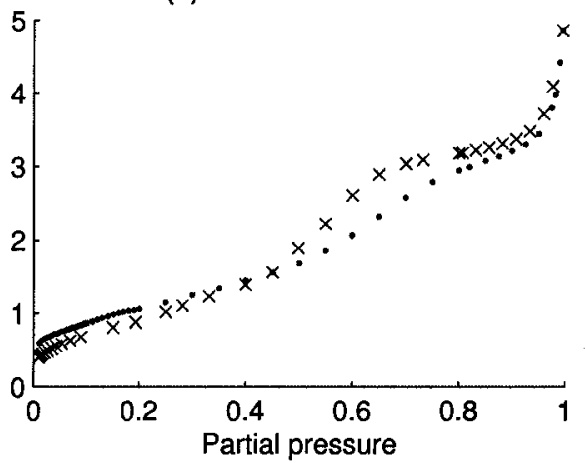

Figure 4.8: BET nitrogen isotherms. Sputtering pressure (a) 2.5 mTorr, (b) 8 mTorr, (c) 25 mTorr, d) 25 mTorr annealed. 


\begin{tabular}{|l|l|l|}
\hline Pressure & As Oxidized $\left(\mathrm{m}^{2}\right)$ & Annealed $300^{\circ} \mathrm{C} 10$ hour $\left(\mathrm{m}^{2}\right)$ \\
\hline 2.5 & 487 & 197 \\
\hline 8.0 & 459 & 215 \\
\hline 25 & 397 & 252 \\
\hline
\end{tabular}

Table 4.2: Surface area of NST normalized to $1 \mathrm{~m}^{2}$ of substrate. Pressure is measured in mTorr.

hysteresis at a relative pressure of about 0.4 , and large volumes of adsorbed nitrogen at extremely low partial pressures. This indicates the presence of both meso-pores and micro-pores respectively [31]. Variations in the pore size distribution with deposition pressure results in films deposited at the lower pressures losing the most surface area post anneal.

Figure 4.8(d) shows the BET isotherm of the same NST film shown in $4.8 \mathrm{c}$ after annealing at $300^{\circ} \mathrm{C}$ for 10 hours. The annealed isotherm shows a significant loss in micro-porosity indicated by the change in the volume of adsorbed nitrogen at the lowest pressure. This explains the significant change in surface area after annealing [31]. Table 4.2 summarizes the results of the surface area analysis. All of the values have been normalized to $1 \mathrm{~m}^{2}$ of substrate.

To properly compare the NST discussed in this paper to other porous high surface area materials $[22,29]$ specific surface area (SSA) measurements in units of $\mathrm{m}^{2} / \mathrm{g}$ are required. The density of the NST film was calculated using the technique in section 4.1.2. Illustrated in Fig. 4.9 are mechanically polished FE-SEM cross-sections of a titanium film sputtered at $25 \mathrm{mTorr}$, the remaining metal, resulting NST after oxidation and annealing.

The thicknesses of the original and remaining Titanium films were measured from 


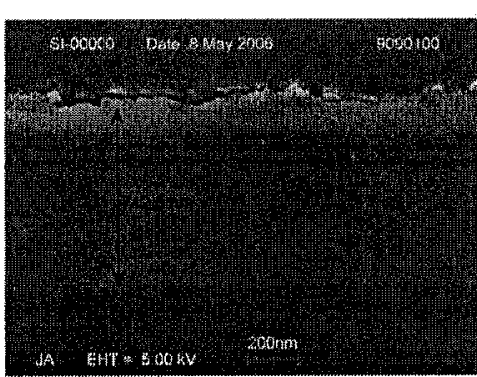

(a)

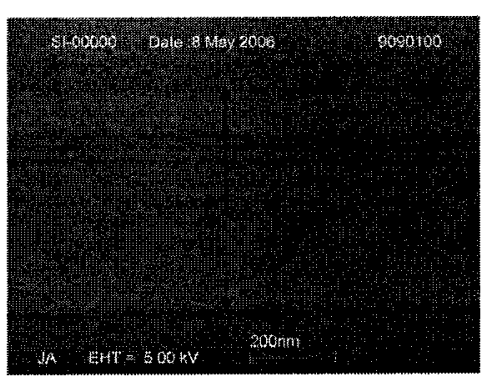

(b)

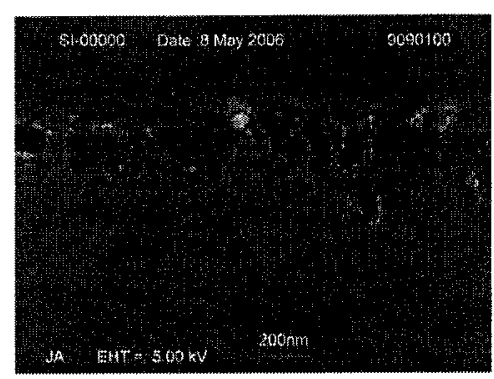

(c)

Figure 4.9: Polished FE-SEM sections of a) parent metal film, b) remaining metal film after oxidation and c) resulting NST

the FE-SEM images, as was the thickness of the resulting NST layer. Equations 4.1 and 4.2 were then used to determine the density of the oxide. The density calculations are summarized in table 4.3 .

\begin{tabular}{|l|l|l|l|l|l|}
\hline Pressure & $M_{a q}$ & $M_{i}$ & $M_{r}$ & $V_{o x}$ & $\rho_{o x}$ \\
\hline 25 & $5.25 e^{-4}$ & $3.2 e^{-3}$ & $2.40 e^{-3}$ & $8.45 e^{-10}$ & $5.42 e^{5}$ \\
\hline
\end{tabular}

Table 4.3: Density measurements of NST film from parent Titanium film deposited at $25 \mathrm{~m}$ Torr on silicon substrate. All of the mass calculations are in grams and density is in $g / \mathrm{m}^{3}$. Pressure and $\mathrm{V}_{o x}$ are measured in $\mathrm{m}$ Torr and $\mathrm{m}^{3}$ respectively

$$
\begin{aligned}
M_{o x} & =A * t_{o x} * \rho_{o x} \\
S S A & =\frac{S A}{M_{o x}}
\end{aligned}
$$

Once the density of the NST standard had been established, the specific surface 
area corresponding to the BET isotherms in Fig. 4.8(d) was calculated with Eq. 4.3. $A$ is the known area of the two four inch wafers and $t_{o x}$ is the thickness of the NST film as measured by SEM cross-section after the surface area analysis was completed. The specific surface area was calculated to be $535 \mathrm{~m}^{2} / \mathrm{g}$. To the best of our knowledge this is the largest measured value of specific surface area for a nanostructured titania thin film reported in the literature [22].

The measured density of the NST layer can be used to calculate the porosity. The porosity $\mathrm{P}$ is defined as:

$$
P=1-\frac{\rho_{p}}{\rho}
$$

Where $\rho$ is the density of bulk anatase $\left(4507 \mathrm{~kg} / \mathrm{m}^{3}\right)$ and $\rho_{p}$ is the density of the NST. Substituting the density measurement from table 4.3 into equation 4.4 reveals that the NST is 85 percent porous.

\subsubsection{Transparency}

Transparency measurements shown in Fig. 4.10 of annealed NST films on glass substrates before dyeing show that the films are highly transparent to visible and infrared light. The strong absorption in the UV is expected for titania. Very little interference between the glass substrate and the NST film is evident from the transmission data, indicating that the refractive index of the titania is probably close to 1.5 (that of glass). This is expected because of the highly porous nature of the NST. Also presented is the transparency of the substrate after soaking in $1 \times 10^{-4} \mathrm{M}$ eosin-y for 24 hours. After soaking in the dye the substrates were rinsed in pure ethanol and stored in the dark. The plot shows a substantial absorption peaking at around 550 
$\mathrm{nm}$. This demonstrates that the NST films have sufficient surface area to acquire a significant dye monolayer, ideal for dye-sensitized solar cells. For comparison the transparency of the glass substrate is presented. The glass appears to be slightly more transparent across the entire spectrum. This is most likely due to scattering in the NST film, which is largely wavelength independent.

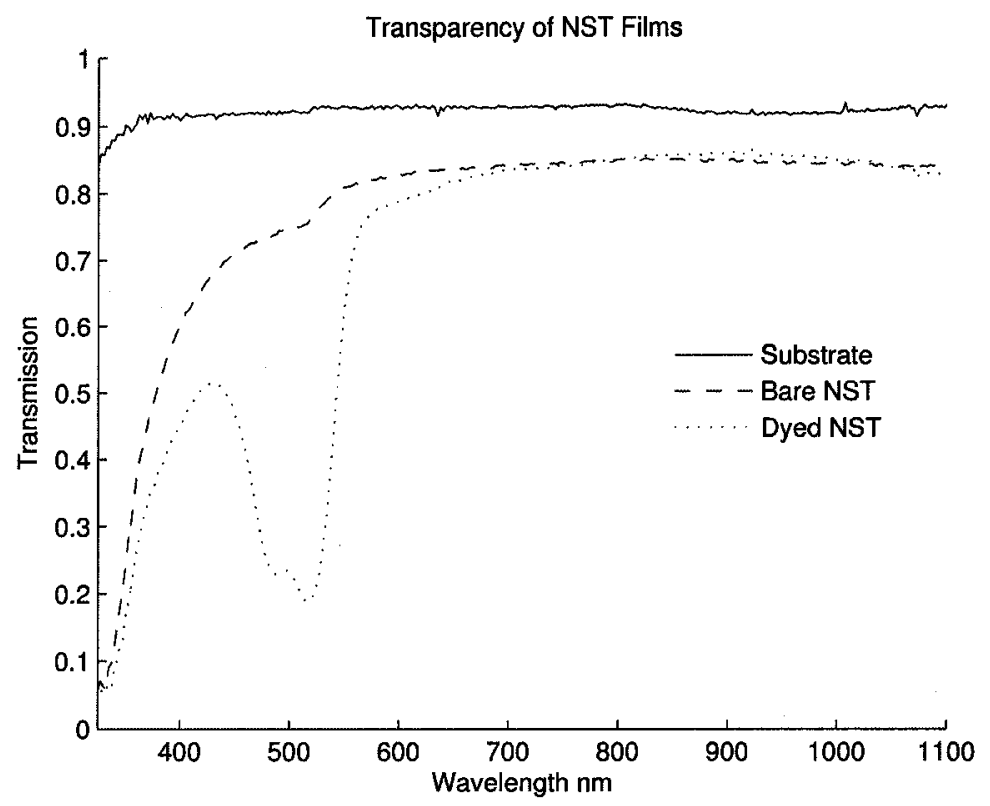

Figure 4.10: Transparency of annealed (bare) and dyed NST films compared to glass substrate

\subsubsection{Xray diffraction measurement}

Figure 4.11 shows the XRD pattern for NST films as-oxidized, annealed for 24 hours at $240^{\circ} \mathrm{C}$ and annealed at $300^{\circ} \mathrm{C}$ for 10 hours. The results demonstrate that a well crystallized anatase phase can be obtained by annealing at temperatures as low as 
$240^{\circ} \mathrm{C}$.

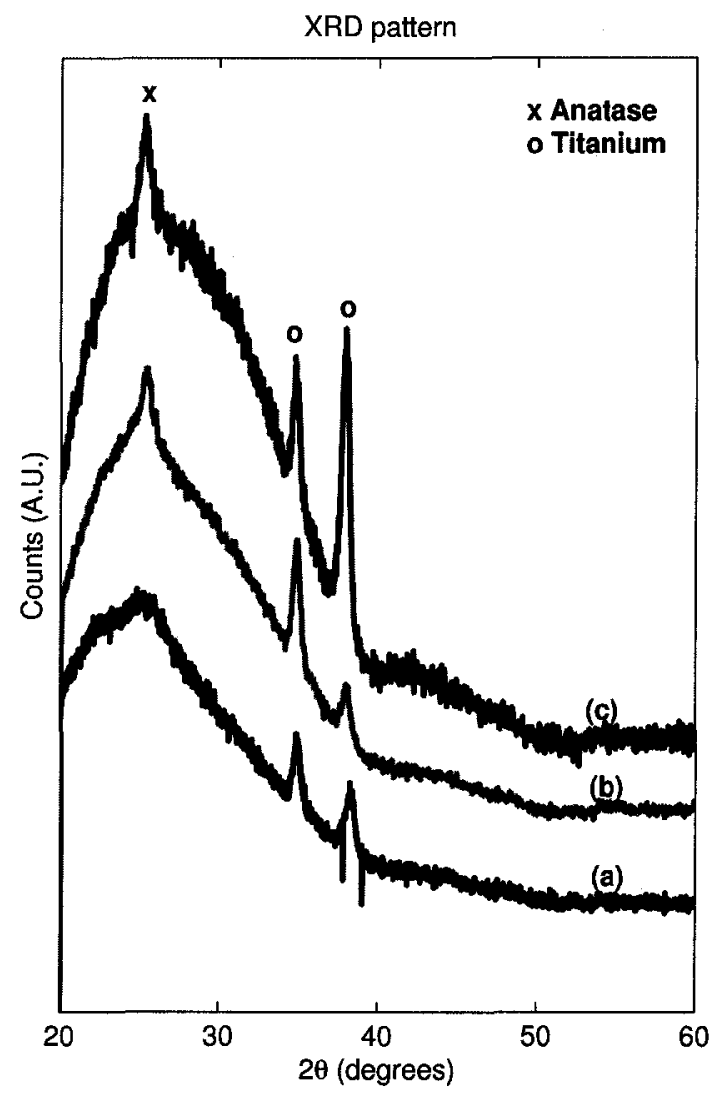

Figure 4.11: XRD pattern of (a) as-oxidized, (b) NST annealed for 24 hours at $240^{\circ} \mathrm{C}$ and (c) 10 hours at $300^{\circ} \mathrm{C}$.

\subsection{Summary}

NST was produced by reacting $10 \%$ hydrogen peroxide with sputtered titanium films. Nitrogen isotherms revealed large surface areas that could be controlled by the deposition pressure. The as oxidized NST appeared to be both meso and micro-porous, 
while the annealed films were more meso-porous. After annealing, the specific surface area of the films was calculated to be nearly $535 \mathrm{~m}^{2} / \mathrm{g}$. To the best of our knowledge this is the largest reported specific surface area for an NST thin film [22, 29]. Parent Titanium films sputtered at lower deposition pressures resulted in NST with more micro-porosity, and therefore higher surface area, but which is significantly reduced when annealed. Transparency measurements of the NST before and after dyeing were presented which demonstrated their potential use in dye-sensitized solar cells.

FE-SEM cross-section presented demonstrated the existence of a previously unreported intermediate layer between the residual metal and the NST film. It was suggested that the intermediate layer was a hydrated titania layer, either a gel or a Lamellar clay. FT-IR measurements revealed the presence of $\mathrm{OH}$ bond stretching in as-oxidized films, but not annealed films, confirming the existence of a hydrated titania layer. It was found that fine tuning the process pressure of the titanium deposition can significantly reduce crack formation in the NST, despite increasing tensile forces in the parent Titanium film. This suggests that although the initial stress in the parent film is not the primary reason for crack formation of the NST, the deposition pressure can be used to control film cracking.

The results presented here have shown that inhibition of crack formation and postanneal surface area were both maximized at the highest available process pressure of 25 mTorr. XRD measurements have shown that an anatase phase could be obtained by annealing at temperatures as low as $240^{\circ} \mathrm{C}$. 


\section{Chapter 5}

\section{Conclusion}

This thesis reports on the production of nanostructured materials NPA and NST thin films using simple chemical and electrochemical means. Nanostructured materials exhibit many unique properties which can be challenging to measure or quantify. Presented here is a detailed analysis of some of these unique properties, as the pertain to NPA and NST.

NPA films are grown by anodically oxidizing $500 \mathrm{~nm}$ of electron beam evaporated aluminium on corning 7509 glass substrates. The aluminium thin films are anodized at $30,40,50,55,70,80$ and $90 \mathrm{~V}$ in a $5 \%$ phosphoric acid solution. The NPA films are highly porous on the nanometer scale. The final thickness of the resulting alumina films after complete conversion of the aluminium metal appears to vary linearly with voltage in the range explored.

Several optical models are suggested and tested for the anodic NPA films. The optical models are fit to transparency data using the LM algorithm. The optical model which best fit the data consisted of a thick oxide, a thin oxide metal mixture 
and a glass substrate. The optical constants of the oxide layer were modeled with a Cauchy approximation. Those of the metal oxide mixture were modeled with a Bruggemann EMA.

Optical measurements for NPA films formed between 30 and $90 \mathrm{~V}$ revealed that the refractive index ranged between about 1.3 and 1.4. The optical model and refractive index measurements could be instrumental in designing optically based sensors. The refractive index increased quickly with voltage until $70 \mathrm{~V}$, at which point it leveled off at just over 1.4. The dependance of refractive index with voltage suggests that contrary to Eq. 3.13 the porosity of the NPA also varies with voltage. It was suggested that this may be due to the microscopic structure of the parent aluminium film, which becomes less important as the pore size increases.

NST films are fabricated using a simple chemical technique first presented in [19]. Titanium films were deposited by RF planar sputtering on silicon and glass substrates. The titanium films were oxidized with heated hydrogen peroxide solution. The resulting NST was highly ordered, porous and amorphous. XRD measurements revealed that annealing the NST at $300^{\circ} \mathrm{C}$ for 10 hours or $240^{\circ} \mathrm{C}$ for 24 hours produced a well crystalized anatase phase. BET surface area analysis revealed that the as oxidized and annealed NST films were high surface area materials. Further studies found that the surface area could be controlled by the deposition pressure of the parent Titanium film. Metal films deposited at $25 \mathrm{mTorr}$ were found to result in NST with the largest post anneal surface area, and so the SSA of such films was measured through an indirect mass measurement. The films are found to be nearly $85 \%$ porous and the SSA was found to be $535 \mathrm{~m}^{2} / \mathrm{g}$. To the best of our knowledge, this is the largest SSA for a titania thin film. 
Studies into the stress of the parent Titanium films as a function of deposition pressure lead to a method where large sheets of crack free NST could be produced, as opposed to the $20 \mu \mathrm{m}$ arrays of NST presented in the literature [5, 19]. FE-SEM imaging and FT-IR analysis suggest that the as-oxidized NST is composed of an amorphous titania layer as well as a hydrated titania layer. Loss of water in the hydrated titania layer due to evaporation after removal from the peroxide solution is thought to be the cause of the cracking observed here and in the literature $[5,19]$.

Transparency measurements of the NST films on glass substrates demonstrate that the films are highly transparent, and also confirm that they are quite porous. Dyeing the NST with eosin-y (Aldrich) demonstrates that it is capable of acquiring a significant dye monolayer. The NST films have been previously demonstrated as being useful in gas sensing devices $[5,19]$. The research presented here suggests that they might also be appropriate in the production of flexible dye sensitized solar cells on heat treated polyimide or polyester substrates. 


\section{Appendix A}

\section{Levenberg-Marquardt algorithm}

The LM algorithm is a popular algorithm for minimizing Eq. 3.1 [32]. Presented here is a brief summary of the algorithm. For a more detailed treatment please see [32].

Equation 3.1 can be rewritten as

$$
F(\mathbf{x})=c \Sigma_{i}^{N}\left[f_{i}-f_{i}^{\prime}(\mathbf{x})\right]^{2}
$$

Where $\mathbf{x}$ is a vector of parameters, $f_{i}$ and $f_{i}^{\prime}(\mathbf{x})$ are the $\mathrm{i}^{\text {th }}$ model and the measured data respectively, $N$ is the number of data points and $c$ is a constant.

To find the best fit for the model, the LM algorithm attempts to minimize the derivative of Eq. A.1 with respect to each of the parameters in $\mathbf{x}$. The derivative is given by

$$
\frac{d F}{d \mathbf{x}}=c \Sigma_{i}^{N}\left[f_{i}-f_{i}^{\prime}\right] \frac{d f_{i}^{\prime}}{d \mathbf{x}}
$$

The jacobian of Eq. A.2 neglecting the $2^{\text {nd }}$ derivative term is given by 


$$
\mathbf{J}=\left[\begin{array}{ccc}
\frac{d F_{1}^{2}}{d x_{1} d x_{1}} & \cdots & \frac{d F_{1}^{2}}{d x_{A} d x_{1}} \\
\cdot & \cdot & \cdot \\
\cdot & \cdot & \cdot \\
\cdot & \cdot & \cdot \\
\frac{d F_{N}^{2}}{d x_{1} d x_{A}} & \cdots & \frac{d F_{N}^{2}}{d x_{A} d x_{A}}
\end{array}\right]
$$

Where $N$ is the total length of the data set and $A$ is the total number of parameters in the model. An initial guess at the vector $\mathbf{x}$ is selected and Eq. A.4 is solved.

$$
\mathbf{x}_{\text {next }}=\mathbf{x}_{\text {last }}-\left[\mathbf{J}^{T} \mathbf{J}+\lambda \mathbf{I}\right] / \mathbf{J}^{T} \frac{d \mathbf{F}}{d \mathbf{x}}
$$

$\lambda$ is a scalar multiplier. At each pass of the algorithm a step is taken for each of the parameters along the gradient of Eq. A.2. The magnitude of $\lambda$ will control their rate of decent along the gradient. At each pass of the algorithm the MSE given by Eq. A.1 is re-evaluated. If the value of MSE has increased since the last pass, the $\mathbf{x}_{n e w}$ values are rejected and the magnitude of $\lambda$ is increased by a factor of 10 , slowing the descent along the gradient. If the new value of MSE is smaller then the $\mathbf{x}_{\text {new }}$ values are accepted and $\lambda$ is decreased by a factor of 10 , increasing the rate of descent.

A Matlab program implementing the LM algorithm was used to extract the refractive index and extinction coefficient from the spectrophotometer data. A set of initial guesses for the Cauchy parameters and the volume fractions $f_{a}$ and $f_{b}$ from Eq. 3.2 and 3.3 were chosen and the LM regression algorithm was used to fit the 
optical model to the measured data. When the LM algorithm had reached a minimum the refractive index and extinction coefficient could be plotted as a function of wavelength using Eq. 3.2. The Matlab program can be found in appendix B. 


\section{Appendix B}

\section{LM Implementation}

\section{B.1 Main program}

\%\%\%\%\%\%\%\%\%\%\%\%\%\%\%\%,LEVEnBERG ALGORITHM MAIN PROGRAM\%\%\%\%\%\%\%\%\%\%\%\%\%\%\%

\% The following program reads in transmission data from a

$\%$ text file and carries out the levenberg algorithm.

$\%$ The MSE and its derivative are calculated using several

$\%$ function calls that defined later

$\%$ Global variables

global L

$\%$ wavelength in microns

global $\mathrm{N} 1 \mathrm{Nm} \mathrm{N3}$

$\%$ refractive index of air, Aluminium metal

$\%$ and glass substrate respectively

\% local variables

$\%$ MSE: mean square error

$\%$ MSE_LAST: previous MSE

$\%$ MSE_NEXT: next MSE

$\% \mathrm{~F}$, Fnext: Derivative of MSE 
\% A: parameters

$\%$ set: parameters to be varied

$\%$ s: selector matrix

try

$\% \% \% \% \% \% \% \% \% \% \% \% \% \% \% \%$ begin input data\%\%\%\%\%\%\%\%\%\%\%\%\%\%\%\%\%\%\%\%\%\%\%\%\%\%\%\%

$\%$ input transmission and refractive index data from a text file

if DATA

$P=$ dlmread ('transmission data.txt');

$\mathrm{L}=\mathrm{P}(:, 1)$;

$\%$ Wavelength (microns)

$\mathrm{P}=\mathrm{P}(:, 2)$;

$\%$ Transmission

$\operatorname{plot}(\mathrm{L}, \mathrm{P})$;

[n $\mathrm{k}]=\mathrm{Al}$;

$\%$ Refractive index of aluminium stored in matlab function $\mathrm{Nm}=\mathrm{n}-\mathrm{j} * \mathrm{k}$;

$\%$ Complex refractive index of aluminium

$\mathrm{N} 1=1$;

$\%$ Refractive index of air

N3 = substrate;

$\%$ Refractive index of substrate stored in matlab function clear $\mathrm{n} \mathrm{k}$;

end

$\% \% \% \% \% \% \% \% \% \% \% \% \% \% \% \% \% \% \%$ end data input $\% \% \% \% \% \% \% \% \% \% \% \% \% \% \% \% \% \% \% \% \% \% \%$

$\% \% \% \% \% \% \% \% \% \% \% \% \% \% \% \% \% \% \% \% \% \%$ Levenberg Algorithm\%\%\%\%\%\%\%\%\%\%\%\%\%\%\%\%\%

$\%$ Initial guesses of parameters

$A=\left[\begin{array}{llllllllllllll}a & \text { a1 } & \text { a2 } & \text { bo } & b 1 & b 2 & 0 & 0 & 0 & 0 & \text { s } & f 1 & \mathrm{df} & \mathrm{dm}\end{array}\right]$;

$\%$ ao - b2 are Cauchy parameters

$\% \mathrm{~s}, \mathrm{f} 1, \mathrm{dm}$ and $\mathrm{df}$ are the scattering coefficient, porosity,

$\%$ oxide thickness and thickness of the mixture layer

set $=[]$;

$\%$ indices of A which represent parameters to be varied in LM 


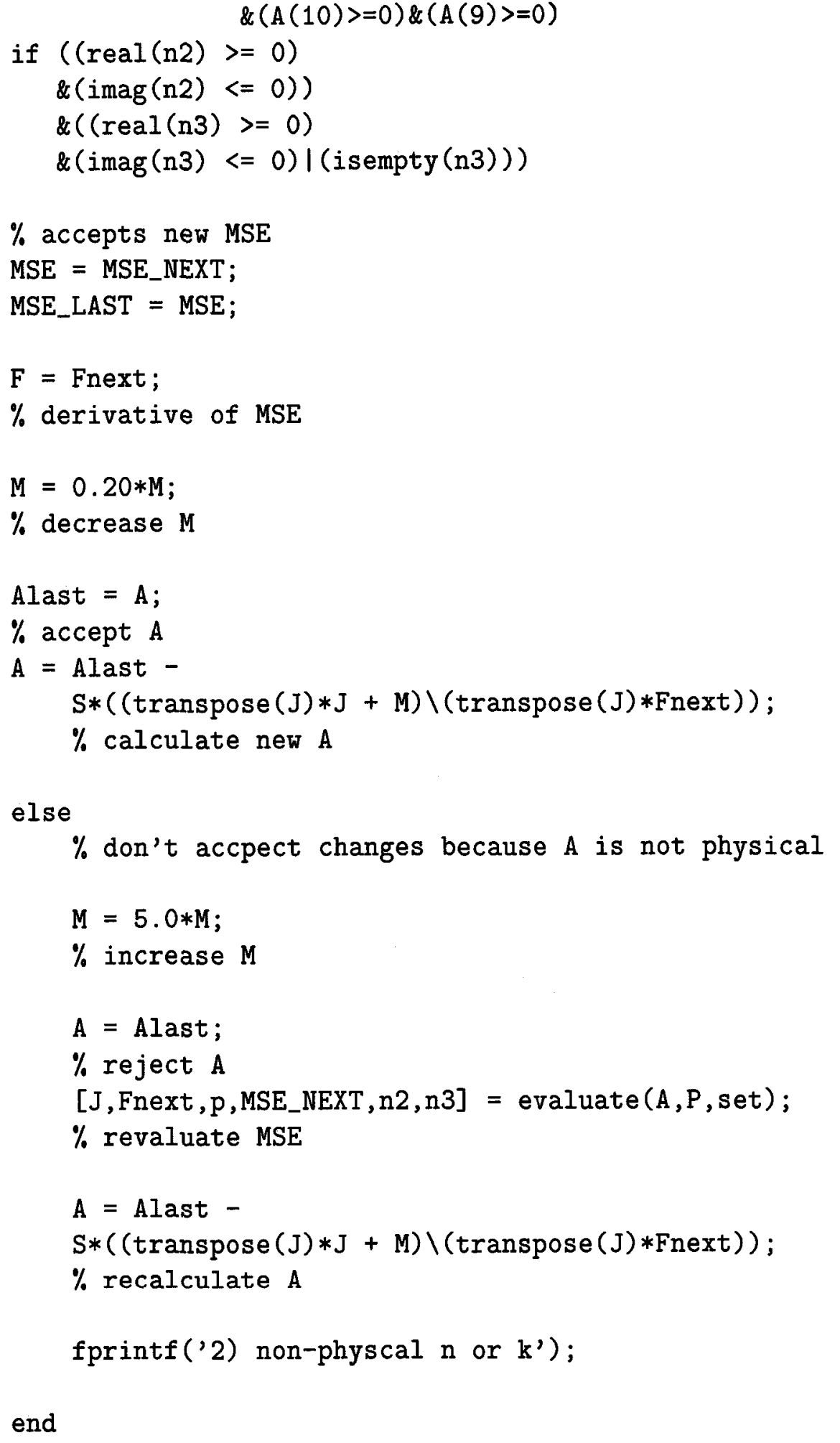


else

$\%$ don't accept changes because

$\%$ MSE_NEXT is larger than MSE

$\mathrm{M}=5.0 * \mathrm{M}$;

$\%$ increase $\mathrm{M}$

$A=$ Alast;

$\%$ reject $A$

$\left[J\right.$, Fnext $\left., p, M S E \_N E X T, n 2, n 3\right]=\operatorname{evaluate}(A, P$, set $)$;

$\%$ recalculate MSE with new $M$

$A=$ Alast -

$\mathrm{S} *((\operatorname{transpose}(\mathrm{J}) * \mathrm{~J}+\mathrm{M}) \backslash(\operatorname{transpose}(\mathrm{J}) *$ Fnext $))$;

$\%$ recalcuate $A$

end

else

$\%$ if this is the first pass of the algorithm proceed here

$\operatorname{try}$

$\left[J\right.$, Fnext $\left., p, M S E \_N E X T, n 2, n 3\right]=$ evaluate $(A, P$, set $)$; catch

lasterr

end

Alast $=A$;

$A=$ Alast -

$\mathrm{S} *((\operatorname{transpose}(\mathrm{J}) * \mathrm{~J}+\mathrm{M}) \backslash(\operatorname{transpose}(\mathrm{J}) *$ Fnext $))$;

$F$ = Fnext;

MSE_LAST $=$ MSE;

MSE $=$ MSE_NEXT;

end

pass $=$ pass +1 ; 


\author{
$\%$ increment pass number \\ end
}

\title{
B.2 evaluate
}

$\%$ This function evaluates the MSE and its first derivative

$\%$ for the main program. The first derivative is evaluated

$\%$ by calling the function 'differentiate' and an optical model.

function $[J$, Fnext $, \mathrm{p}, \mathrm{MSE}, \mathrm{n} 2, \mathrm{n} 3]=\operatorname{evaluate}(\mathrm{A}, \mathrm{P}$, set $)$

$\%$ variables

global L \%wavelength

$\% \mathrm{~A}=$ parameters

$\% \mathrm{P}=$ data

$\%$ set = parameters to be varied

$\operatorname{par}=\mathrm{A}$;

fun $=$ QthinfilmTA12; \%name of function which contains optical model

for $i=1:$ length (set)

try

inext $(:, i)=\operatorname{differentiate}($ fun, $\operatorname{par}, \operatorname{set}(i), \operatorname{length}(L))$;

$\%$ numerically determines the derivatives of the MSE

$\%$ with respect to the parameters being varried catch

lasterr

return

end

end

$\%$ returns the refractive index of the model

$[p, n 2, n 3]=\operatorname{fun}([], \operatorname{par},[], 1)$;

Fnext $=\quad$-inext'*(P-p); J = transpose (inext) $*$ inext;

MSE $=\quad \operatorname{sum}\left((P-p) \cdot{ }^{-2}\right) /($ length $(L)-$ length $(A)) ;$ 


\section{B.3 differentiate}

\% This function evaluates the derivative of the MSE

$\%$ by calling the function which contains the optical model

function $d=$ differentiate (fun,par, set,s)

$\%$ vari varies the parameter by a small amount

$\%$ the model is evaluated at each variation of the parameter

$\operatorname{vari}=[\operatorname{par}($ set $)+50000 *$ eps $\operatorname{par}($ set $)-50000 * e p s]$;

$\mathrm{p}=\operatorname{zeros}(\mathrm{s}, 2)$

for $m=1: 2$

try

$p(:, m)=$ fun $(\operatorname{vari}(m)$, par, set, 0$) ;$

catch

lasterr

return

end

end

\%evaluates the change in the model for the small variation

$\%$ in the paratmeter

$d=\operatorname{diff}\left(p^{\prime}\right) /(2000 * e p s) ; d=-d^{\prime}$;

\section{B.4 thinfilmTAl2}

$\%$ This function evaluates the refractive index

$\%$ and the modeled transmission data for the main program.

$\%$ It contains the optical model.

function $[p, N f, n 3]=$ thinfilmTAl2(vari,par, set,MSE) global L $\%$ wavelength

global N1 N3 Nm

$\%$ refractive index of the air, substrate and metal 
$\%$ defines the parameters

$A 0=\operatorname{par}(1) ; A 1=\operatorname{par}(2) ; A 2=\operatorname{par}(3) ; B o=\operatorname{par}(4) ;$

$\mathrm{B} 1=\operatorname{par}(5) ; \mathrm{B} 2=\operatorname{par}(6) ; \mathbf{s}=\operatorname{par}(11)$;

$f 1=\operatorname{par}(12) ; \mathrm{df}=\operatorname{par}(13) ; \mathrm{dm}=$ $\operatorname{par}(14)$;

$\%$ if the function was called to evaluate the

$\%$ derivative proceed here to set which parameter

$\%$ is to be varied

if $\sim \mathrm{MSE}$

if set $==1$

Ao $=\operatorname{var} i$

else if set $==2$

$A 1=$ vari;

else if set $==3$

A2 = vari

else if set $==4$

Bo $=\operatorname{vari}$;

else if set $==5$

$B 1=\operatorname{var} i$

else if set $==6$;

$B 2=\operatorname{var} i$;

else if set $==9$

$f 2=\operatorname{var} i$

else if set $==10$

$f 3=\operatorname{var} i$;

else if set $==11$;

$\mathrm{s}=\operatorname{vari}$

else if set $==12$;

$f 1=\operatorname{var} i$;

else if set $==13$;

$\mathrm{df}=\operatorname{var} i$;

else if set $==14$;

$\mathrm{dm}=\operatorname{var} i$

end

end

end

end 


$$
\begin{aligned}
& \text { end } \\
& \text { end } \\
& \text { end } \\
& \text { end } \\
& \text { end } \\
& \text { end } \\
& \text { end } \\
& \text { end } \\
& \text { end }
\end{aligned}
$$

\%\%\%\%\%\%\%\%\%\%\%\%\%\%\%\%\%\%\%\%\%\%\%mode1\%\%\%\%\%\%\%\%\%\%\%\%\%\%\%\%\%\%\%\%\%\%\%\%\%\%\%\%

$\% \mathrm{n}$ and $\mathrm{k}$ for the oxide are defined in

$\%$ terms of the Cauchy paramters

$\mathrm{n}=\mathrm{Ao} \cdot{ }^{\wedge} 2+\mathrm{A} 1 \cdot{ }^{-2} \cdot / \mathrm{L} \cdot{ }^{-2}+\mathrm{A} 2 \cdot{ }^{-2} \cdot / \mathrm{L} \cdot{ }^{-4}$;

$\mathrm{k}=\mathrm{Bo} \cdot{ }^{\wedge} 2+\mathrm{B} 1 \cdot{ }^{\wedge} 2 \cdot / \mathrm{L} \cdot{ }^{-} 2+\mathrm{B} 2 \cdot{ }^{-2} 2 / \mathrm{L} \cdot{ }^{-4} ; \mathrm{Nf}=\mathrm{n}-\mathrm{j} * \mathrm{k}$;

$\%$ complex index of refraction for the oxide

ef $=(N f) \cdot{ }^{-2}$;

$\%$ permittivity of oxide

$\mathrm{em}=(\mathrm{Nm}) \cdot{ }^{-2}$;

$\%$ permittivity of metal

$\%$ solves the bruggeman EMA

$A=(1-f 1 . \wedge 2) *(1-($ ef./em $)) . /(($ ef. $/$ em $) . \wedge(1 / 3)) ;$

$B=\left(0.5 *\left(1+(1+(4 * A . \wedge 3) / 27) \cdot{ }^{\wedge} 0.5\right)\right) \cdot{ }^{\wedge}(1 / 3)$;

eff $=(1-A . * B+A \cdot \wedge 2 \cdot /(3 * B)) . * e m$;

Neff $=\operatorname{sqrt}($ eff $)$;

$z=0.0027$

$\%$ impedance of space

$\%$ optical transfer function of thin film structure

$\mathrm{Y} 1=\mathrm{z} * \mathrm{~N} 1 ; \mathrm{Yf}=\mathrm{z} * \mathrm{Nf} ; \mathrm{Ym}=\mathrm{z} * \mathrm{Neff} ; \mathrm{Ys}=\mathrm{z} * \mathrm{~N} 3$;

$\mathrm{Bf}=2 * \mathrm{pi} * \mathrm{Nf} * \mathrm{df} ; \mathrm{Bm}=2 * \mathrm{pi} * \operatorname{Neff} * \mathrm{dm}$

for $i=1:$ length $(L)$ 


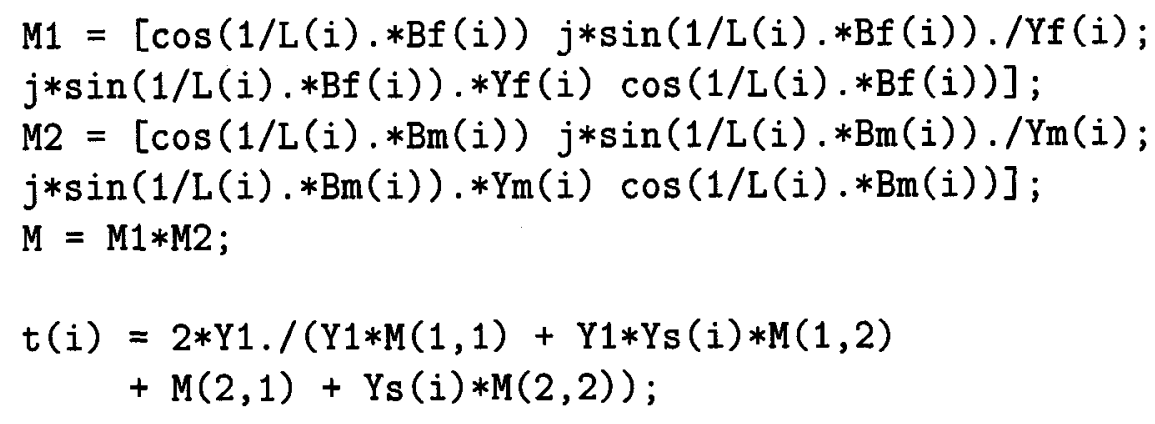

end

$\%$ returns the modeled transmission data

$\mathrm{p}=\mathrm{s} * \mathrm{~N} 3 . *\left(\operatorname{abs}(\mathrm{t}) \cdot{ }^{-2}\right)^{\prime}$; 


\section{References}

[1] M. Gratzel, "Photoelectrochemical cells," Nature, vol. 414, 2001.

[2] S. Z. Chu, K. Wada, S. Inoue, and S. Todoroki, "Formation and microstructures of anodic alumina films from aluminium sputtered on glass substrate," Journal of the Electrochemical Society, vol. 149, no. 7, 2002.

[3] A. P. Li, F. Muller, A. Briner, K. Nielsh, and U. Gosele, "Hexoganal pore arrays with a $50-420 \mathrm{~nm}$ interpore distance formed by self-organization in anodic alumina," Journal of Applied Physics, vol. 84, no. 11, 1998.

[4] A. Zuruzi and N. MacDonald, "Facile fabrication and integration of patterned nanostructured tio2 for microsystems applications," Adv. Funct. Mater., vol. 15, no. 3,2005 .

[5] A. S. Zuruzi and B. C. Butler, "Integrating biomaterials into microsystems: Formation and characterization of nanostructured titania," Mat. Res. Soc. Symp. Proc, vol. 820, 2004.

[6] Z. A. Samah, A. Kolmakov, M. Moskovits, and N. C. MacDonald, "Low cost integrated utilizing patterned nano-structred titania arrays fabricated using a simple process," Mat. Res. Soc. Symp. Proc, vol. 828, 2005. 
[7] M. Gratzel, "Conversion of sunlight to electric power by nanocrystalline dyesensitized solar cells," Journal of Photochemistry and Photobiology A: Chemistry, vol. 164, pp. 3-14, 2004.

[8] G. L. Hornyak, C. J. Patrissi, and C. R. Martin, "Fabrication, characterization and optical properties of gold nanopoarticle/porous alumina composites: The nanoscattering maxwell-garnett limit," Journal of Physical Chemistry B, vol. 101, pp. 1548-1555, 1997.

[9] S. K. Thamida and H.-C. Chang, "Nanoscale pore formation dynamics during aluminium anodization," Chaos, vol. 12, no. 1, 2002.

[10] J. W. Diggle, T. C. Downie, and C. W. Goulding, "Anodic oxide films on aluminium."

[11] K. H. A. Lau, L.-S. Tan, K. Tamada, M. S. Sander, and W. Knoll, "Highly sensitive detection of process occuring inside nanoporous anodic alumina: A waveguide optical study," Journal of Physical Chemistry B, 2004.

[12] S.-Z. Chu, S. Inoue, K. Wada, D. Li, and H. Haneda, "Highly porous tio2/al2o3 composite nanostructures on glass by anodization and the sol-gel process: fabrication and photocatalytic characterisitcs," Journal of Materials Chemistry, 2003.

[13] S.-Z. Chu, S. Inoue, K. Wada, S. Hishita, and K. Kurashima, "Self-orgainzed nanoporous anodic titania films and ordered titania nanodots/nanorods on glass," Advanced Functional Materials, 2005. 
[14] Y. Wang, Z.-H. Jiang, and F.-J. Yang, "Preparation and photocatalytic activity of mesoporous tio2 derived from hydrolysis condensation with tx-100 as template," Materials Science and Engineering B, vol. 128, pp. 229-233, 2006.

[15] C. A. F. Jr, G. L. Hornyak, J. A. Stockert, and C. R. Martin, "Templatesynthesized nanoscopic gold particles: Optical spectra and the effects of particle size and shape," Journal of Physical Chemistry, vol. 98, pp. 2963-2971, 1994.

[16] G. E. Thompson, "Porous anodic alumina: fabrication, characterization and applications," Thin Solid Films, vol. 297, pp. 192-201, 1997.

[17] M. Paulose, K. Shankar, O. K. Varghese, G. K. Mor, B. Hardin, and C. A. Grimes, "Backside illuminated dye-sensitized solar cells based on titania nanotube array electrodes," Nanotechnology, vol. 17, pp. 1446-1448, 2006.

[18] P. G. Miney, P. E. Colavita, M. V. Schiza, R. J. Priore, F. G. Haibach, and M. L. Myrick, "Growth and characterization of a porous aluminium oxide film formed on an electricaly insulating support," Electrochemical and Solid State Letters, vol. 6, no. 10, 2003.

[19] J.-M. Wu, S. Hayakawa, K. Tsuru, and A. Osaka, "Porous titania films prepared from interaction of titanium with hydrogen peroxide solution," Scripta Materialia, vol. 46, 2002.

[20] O. Jessensky, F. Muller, and U. Gosele, "Self-organized formation of hexogonal pore arrays in anodic alumina," Applied Physics Letters, vol. 72, no. 10, 1998.

[21] H. G. Tompkins, Spectroscopic ellipsometry and reflectometry: a users guide, March. 
[22] Y. Ohsaki, N. Masaki, T. Kitamura, Y. Wada, T. Okamoto, T. Sekino, K. Niihara, and S. Yanagida, "Dye-sensitized $\mathrm{tio}_{2}$ nanotube solar cells: fabrication and electronic characterization," Chem. Phys., vol. 7, 2005.

[23] R. Beranek, H. Hildebrand, and P. Schmuki, "Self-organized porous titanium oxide prepared in h2so4/hf electrolytes," Electrochemical and Solid-State letters, vol. 6,2003 .

[24] J. M. Macak, H. Tsuchiya, and P. Schmuki, "High-aspect-ration tio $_{2}$ nanotubes by anodization of titanium," Angew. Chem. Int., vol. 44, 2005.

[25] O. K. Varghese, D. Gong, M. Paulose, and C. A. G. E. C. Dickey, "Crystallization and high-temperature structural stability of titanium oxide nanotube arrays," J. Mater. Res., vol. 18, 2003.

[26] "Labjack-usb/ethernet based data acquistion and control," www.labjack.com.

[27] E. D. Palik, Handbook of optical constants of solids. Academic Press, 1985.

[28] E. Hecht, Optics. Addison-Wesley Pub. Co., 1987.

[29] J. Zhao, Z. Wang, L. Wang, H. Yang, and M. Zhao, "The preparation and mechanism studies of porous titania," Materials Chemistry and Physics, vol. 63, pp. 9-12, 2000 .

[30] P. Tengvall, I. Lundstrom, L. Sjoqvist, and H. Elwing, "Titanium-hydrogen peroxide interaction: model studies of the influence of the inflammatory response of titanium implants," Biomaterials, vol. 10, 1989. 
[31] S. Gregg, Adsorption, surface area, and porosity. London England Academic, 1982.

[32] W. H. Press, Numerical recipies in FORTRAN: the art of scientific computing. Cambridge Univeristy Press, 1992. 